\title{
The preliminary inventory of coseismic ground failures related to December 2020 - January 2021 Petrinja earthquake series
}

\author{
Davor Pollak ${ }^{1, *}$, Vlatko Gulam ${ }^{1}$, Tomislav Novosel ${ }^{1}$, Radovan Avanić ${ }^{2}$, Bruno Tomljenović ${ }^{3}$, \\ Nina Hećej ${ }^{1}$, Josip Terzić ${ }^{1}$, Josip Stipčević ${ }^{4}$, Mario Bačić ${ }^{6}$, Tomislav Kurečić ${ }^{2}$, Mario Dolić ${ }^{1}$, \\ Iris Bostjančić ${ }^{1}$, Lara Wacha ${ }^{2}$, Ivan Kosovići ${ }^{1}$, Marko Budić2 ${ }^{2}$, Matija Vukovski², Nikola Belić2 ${ }^{2}$ \\ Marko Špelić ${ }^{2}$, Vlatko Brčić2, Josip Barbača², Branko Kordić2 ${ }^{2}$, Damir Palenik², Radovan Filjak², \\ Tihomir Frangen ${ }^{1}$, Mirja Pavić ${ }^{1}$, Kosta Urumović ${ }^{1}$, Marin Sečanj ${ }^{7}$, Bojan Matoš ${ }^{3}$, Marin Govorčin ${ }^{5}$, \\ Meho Saša Kovačević 6 and Lovorka Librić6

\footnotetext{
${ }^{1}$ Croatian Geological Survey, Department of Hydrogeology and Engineering Geology, 10000 Zagreb, Croatia; ("corresponding author: dpollak@hgi-cgs.hr)

${ }^{2}$ Croatian Geological Survey, Department of Geology, 10000 Zagreb, Croatia

${ }^{3}$ University of Zagreb, Faculty of Mining, Geology and Petroleum Engineering, Department of Geology and Geological Engineering, 10000 Zagreb, Croatia

${ }^{4}$ University of Zagreb, Faculty of Science, Department of Geophysics, 10000 Zagreb, Croatia

${ }^{5}$ University of Zagreb, Faculty of Geodesy, 10000 Zagreb, Croatia

${ }^{6}$ University of Zagreb, Faculty of Civil Engineering, Department of Geotechnical Engineering, 10000 Zagreb, Croatia

${ }^{7}$ Independent researcher, Katarine Zrinski 6, 10370 Dugo Selo, Croatia
}

doi: $10.4154 / g c .2021 .08$

\section{Article history: \\ Manuscript received January 26, 2021 Revised manuscript accepted March 01, 2021 Available online May 06, 2021}

Keywords: Petrinja, earthquake, ground failures, inventory, dropout dolines, liquefaction, landslides

\begin{abstract}

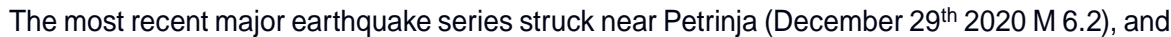
triggered extensive ground failures in the wider area of Petrinja, Sisak and Glina. Coseismic ground failures including subsidence dolines, liquefaction and landslides have been documented over a large area by various experts and teams. These data are stored in the newly created inventory, which is openly presented in this paper. This inventory is administered and updated by the Croatian Geological Survey, and will be available online via a Web Map Service (WMS) (www.hgi-cgs.hr). The aim of the inventory is to not only provide data for the development of susceptibility maps and more detailed exploration for possible remediation measures, but also to define the priorities for immediate action. The earthquake triggered the rapid development of dropout dolines which endanger the local populations of the villages of Mečenčani and Borojevići. This is still an ongoing process in the vicinity of the houses and therefore in-situ exploration started immediately. Liquefaction related to alluvial sediments of the Sava, Kupa and Glina rivers occurred almost exclusively in loose and pure sands, and was accompanied by sand boils, subsidence and lateral spreading. Liquefaction also presents a greater hazard because settlement of houses and river embankments occurred. Lateral spreading caused failures of river flood embankments and natural river banks. According to the data known to date, the majority of the coseismic landslides were reactivated with minor displacements. Despite that, it has been recognised that houses at the edge, or in landslide colluvium suffered greater damage than other houses located outside the landslide impact zone.
\end{abstract}

\section{INTRODUCTION}

Ground shaking during an earthquake usually causes major damage to structures. However, earthquakes also induce ground failures that can significantly contribute to total earthquake loss.

"The term ground failure is a general reference to landslides, liquefaction, lateral spreads, and any other consequence of shaking that affects the stability of the ground" (USGS Earthquake Glossary, 2021).

Unfortunately, numerous cases worldwide witness that not enough attention is directed toward the identification of various types of ground failure which usually follow very strong or severe earthquakes (BIRD \& BOMMER, 2004). The absence of ground failure data may lead to unrealistic projections of losses caused by a particular earthquake. Furthermore, evidence of such processes/phenomena represent an important input into the production of reliable susceptibility maps and microzoning, which are important in the proper evaluation of seismic hazard in a particular region. Most of such databases in the literature are related to landslides (KEEFER, 1984; RODRÍGUEZ et al.,
1999, BOMMER \& RODRÍGUEZ, 2002; GORUM et al., 2011) or liquefaction (YOUD \& HOOSE, 1978), but there are also more recent examples of inventories comprising all the typical forms of ground failure caused by earthquakes (PRESTININZI \& ROMEO, 2000).

It is well known that Croatia lies in a seismically active region of the central Mediterranean, characterized by seismicity that is unevenly distributed across the country, both in the Adriatic (HERAK et al., 2005; HERAK et al., 2017b; GOVORČIN et al., 2020) and in the Pannonian basin area (HERAK et al., 2009; HERAK et al., 2020; MARKUŠIĆ et al., 2020). Historical and instrumentally recorded data indicate the three most seismically active areas are: NW Croatia (greater Zagreb area), the Croatian Primorje (greater Rijeka area) and Dalmatia. They are clearly distinguished on the seismic hazard map for the Republic of Croatia (HERAK et al., 2011) (Figure 1). Since the largest city in Croatia is also situated in a seismically active area, the group of authors have created a database of geotechnical boreholes which contains relevant data for soil behaviour and site response analy- 
ses for the City of Zagreb (KVASNIČKA et al., 1998; KVASNIČKA \& MATEŠIĆ, 2001; MATEŠIĆ \& KVASNIČKA, 2007; MATEŠIĆ et al., 2011).

Although there have been a number of $\mathrm{M}_{\mathrm{L}} \geq 6.0$ earthquakes registered in the last millennium, historical data about earthquake-triggered ground failures in Croatia are scarce and incomplete. As a result, such an inventory does not yet exist. However, the most recent December $29^{\text {th }} 2020$ M 6.2 earthquake near Petrinja, which together with the foreshock on December 28 (M 5.0) and the following aftershock series that is still ongoing, triggered extensive coseismic ground failures and initiated the creation of such inventory. Therefore, the focus of this work is to present the structure of the initial inventory and data on coseismic ground failures collected during first two weeks of the Petrinja earthquake series after the main shock of M 6.2 that occurred on $29^{\text {th }}$ December 2020. The work also indicates the most affected zones, and provide information to a wider audience, the general public, local and regional administrations and Civil Protection for further reference. We also hope that open access to the collected data will motivate other earth-scientists, experts, and the public to provide their contribution to the database, in order to establish the most complete insight into all coseismic ground failures and processes triggered by the Petrinja earthquake series. The database would also enable more realistic estimations of the total earthquake damage and loss.

For this reason, the complete table of inputs collected so far is presented here in order to transparently provide information that can be further used to develop susceptibility maps, identify the most critical surface damage zones and ensure data for seismic microzoning and hazard assessment. Detected coseismic ground failures will be additionally explored in much more detail to provide better insights into local conditions, triggering forces and failure mechanisms, in order to provide parameters for possible remediation measures. The database can also serve as a valuable factor in the development of the design and construction policies for the rehabilitation of the buildings and infrastructure in the area, thus ensuring the long-term safety of residents.

The data presented in this preliminary inventory was mainly collected by geologists from the Croatian Geological Survey (HGI), supplemented by inputs from other earth-scientists and geotechnical engineers from the University of Zagreb (The Faculty of Mining, Geology \& Petroleum Engineering and the Faculty of Science; Faculty of Civil Engineering), other civil engineering experts, local citizens and the media. Therefore, it is important to point out that not all of the entries presented here are verified yet by geologists. In addition, the inventory for this particular earthquake series should be considered as preliminary, since we expect that additional data will be collected over time. The up to date database is presented online via Web Map Service (WMS), and will be continuously maintained and updated by the HGI (www.hgi-cgs.hr).

\section{OVERVIEW OF GEOLOGY AND SEISMICITY OF THE RESEARCH AREA}

\subsection{Geology}

The Petrinja earthquake series (December 2020 - January 2021) took place along the southwestern margin of the Sava Basin of the Pannonian Basin System (PAVELIĆ \& KOVAČIĆ, 2018, SAFTIĆ et al. 2003 with references). A simplified geological map of the earthquake series affected area (the research area) is shown

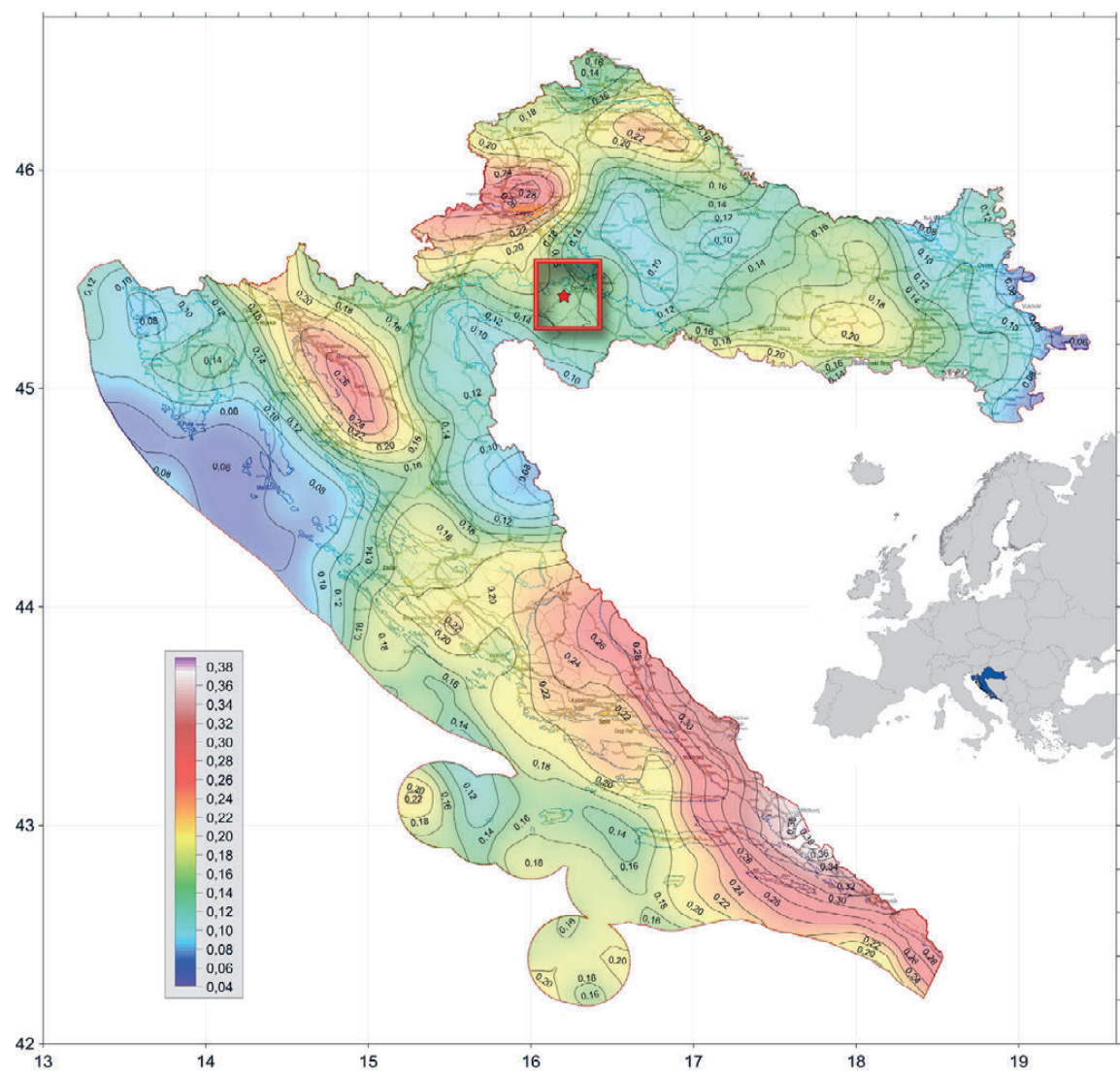

Figure 1. The location of Croatia marked blue on the European map (right). Geographical location of the research area (red rectangle) at the seismic hazard map for the Republic of Croatia (HERAK et al., 2011). Red symbol indicates the location of the earthquake epicentre (29.12.2020). Map shows peak ground acceleration (PGA) for $10 \%$ probability of exceedance in 50 years in units of standard gravity $(\mathrm{g})$ for reference rock soil $(\mathrm{vs} 30=800 \mathrm{~m} / \mathrm{s})$. This corresponds to PGA-values that return on average every 475 years. 


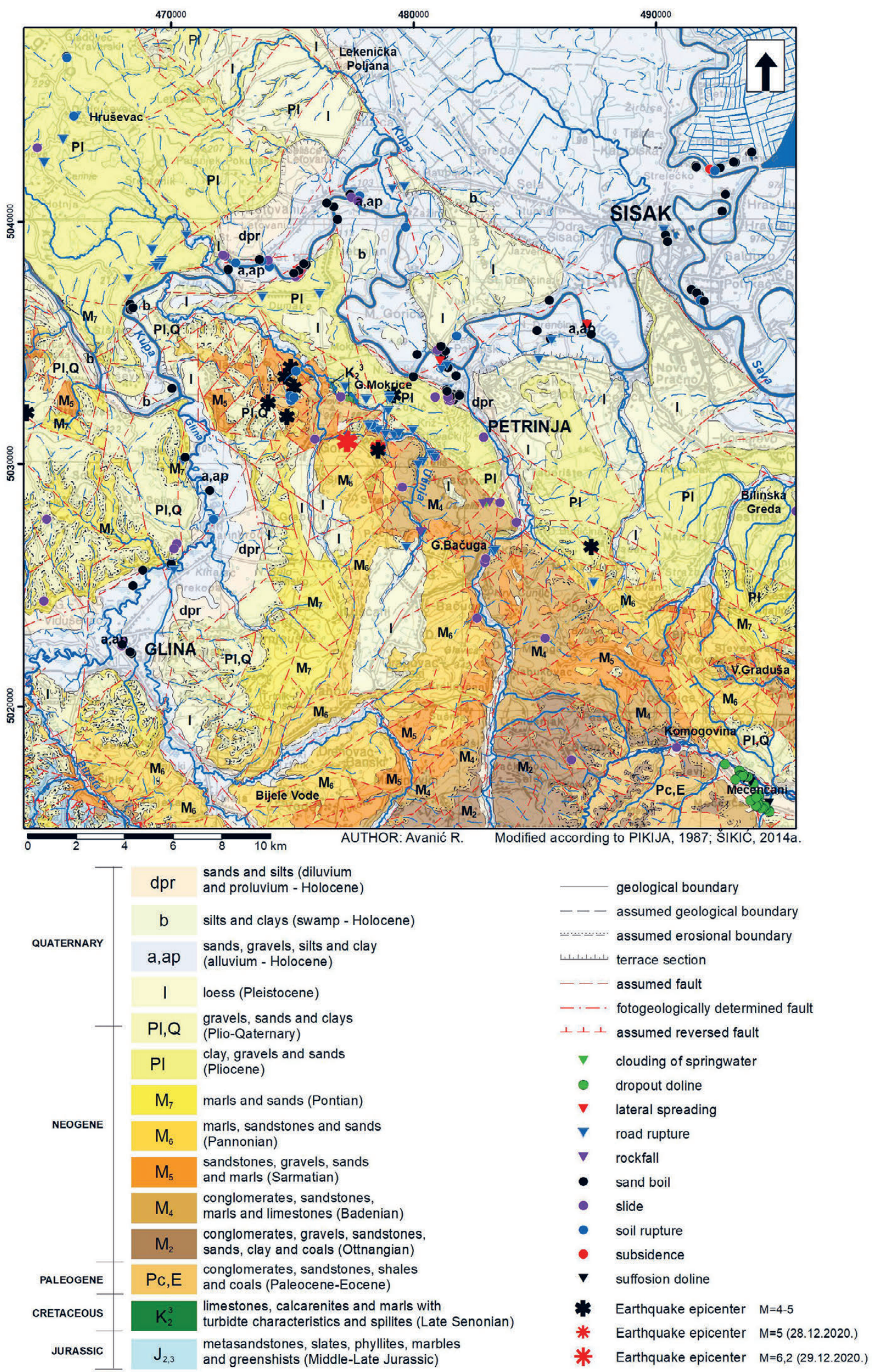

Figure 2. The geological map of the research area (originally in the scale 1:100.000) with the locations of ground failures. 
on Figure 2, modified after the Basic Geological Map of SFRY 1:100.000, sheet Sisak (PIKIJA, 1987) and the Basic Geological Map of the Republic of Croatia 1:100.000, sheet Bosanski Novi (ŠIKIĆ, 2014a).

The research area is mainly covered by Neogene and Quaternary deposits, while older pre-Neogene basement rocks (mostly Palaeogene) are only locally exposed in the SE part of the area (PIKIJA, 1987). In the following text we shall briefly describe the major characteristics of the stratigraphic units presented on the map (Figure 2). Some more details are presented for units that are subjected to ground failure.

The oldest exposed rocks in the research area are of Middle to Late Jurassic age $\left(\mathrm{J}_{2,3}\right)$, represented by metasandstones, slates, phyllites, marbles and greenschists, as well as by metamorphosed magmatic rocks (ŠIKIĆ, 2014b, RAFFAELLI \& MAGDALENIĆ, 1970; MAJER, 1984; MAJER \& LUGOVIĆ, 1985). The thickness of this unit is up to $400 \mathrm{~m}$ (ŠIKIĆ, 2014a). Small outcrops of the Cretaceous sedimentary sequence (Late Senonian $-\mathrm{K}_{2}{ }^{3}$ ) consist of limestones with pyroclastics and radiolarian chert intercalations.

Palaeogene deposits (Palaeocene-Eocene-Pc, E) are mainly conglomerates (Figure 2). The conglomerates are carbonate-free, polymictic with various amounts of sandy matrix. Along with conglomerates, intercalations of sandstones, shales and coal seams, marls and limestones are present. An estimated thickness of these deposits is around $200 \mathrm{~m}$ (ŠIKIĆ, 2014b).

Ottnangian deposits $\left(\mathrm{M}_{2}\right)$ are mostly represented by coarseand fine-grained clastics (ŠIKIĆ, 2014b). Conglomerates, gravels and sands are mostly seen in the lower part of the sequence, while the upper part is composed of an alternation of marls, clays and limestones. The thickness of the Ottnangian clastics is assumed to be $250-300 \mathrm{~m}$.

Between the river Kupa in the northwest and Mečenčani village in the southeast (Figure 2), Badenian deposits $\left(\mathrm{M}_{4}\right)$ are exposed. They unconformably rest over Palaeogene and Ottnangian sediments, above distinctly recognized erosional boundary. In the basal part they are represented by conglomerates, gravels, sandstones and biocalcarenites that grade upward into finergrained clays and marls, and into bioclastic and biogenic limestones in the uppermost part of the sequence (PIKIJA, 1986; ŠIKIĆ, 2014b). The thickness of Badenian sediments is variable, reaching up to $300 \mathrm{~m}$.

Sarmatian deposits $\left(\mathrm{M}_{5}\right)$ continuously follow on top of Badenian deposits. They began with coarse-grained clastics composed of polymictic conglomerates, gravels, sandstones, sandy clays and marls deposited in coastal environments (ŠIKIĆ, 2014b). In deep-water environments of the basin, laminated sands, marls, and limestones prevail. The thickness of the Sarmatian deposits is a maximum of $100 \mathrm{~m}$.

Pannonian clastics $\left(\mathrm{M}_{6}\right)$ are divided into the Lower and Middle Pannonian. The most frequent sediments of the Lower Pannonian are laminated and platy limestones (micrites and clayey micrites), of the Croatica formation, locally interlayered with sands and sandy-marls, and with local occurrences of cm-thick gravel interlayers (ŠIKIĆ, 2014b). In the Glina and Zrin-Dvor valleys, middle Pannonian, laminated to thin-layered sands and sandy marls alternate with clayey limestones, and poorly lithified sandstones. The Croatica fm. is followed by the Medvedski breg $\mathrm{fm}$., which is represented by massive to weakly layered marls and sporadic clayey limestones and clays. Sediments of the Medvedski breg fm. are deposited in the more distal part of the lacustrine basin (AVANIĆ et al., 2018b). The thickness of the Pannonian deposits is between 120 to $200 \mathrm{~m}$.

Pontian deposits $\left(\mathrm{M}_{7}\right)$ are mostly characterized by marls. In the lower part, clayey and sandy marls predominate, while in the upper part, clayey siltstones and sands prevail (ŠIKIĆ, 2014b). In the area of the Glina River, layers of coal-clay seams and interlayers of lignite are found. According to the latest lithostratigraphic nomenclature, Pontian deposits are subdivided into the Andraševec and Hum Zabočki formations, based on a subdivision known in the area of northern Croatia (KOVAČIĆ, 2004; GRIZELJ, 2004). The Andraševec formation is composed of the alternation of sands, calcite-rich silts and marls, deposited since the Late Pannonian to the Late Pontian (AVANIĆ et al., 2018a). It is continuously overlain by the Hum Zabočki formation of the Late Pontian that mostly consists of fine- to medium-grained sands, silty marls, clays and coal seams (cm to dm thick). The thickness of the Pontian deposits varies between 150 to $450 \mathrm{~m}$.

Pliocene deposits $(\mathrm{Pl})$ are composed of coarse- to fine-grained clastics , mostly sands, gravels, silts and clays with a general coarsening upwards trend from the Lower to Upper Viviparus beds (MANDIC et al., 2015; KUREČIĆ, 2017; KUREČIĆ et al., submitted) (Figure 2). Lignite layers are also common. Pliocene sediments are equivalent to the Vrbova fm. of the Sava basin (AVANIĆ et al., 2018a; HALAMIĆ et al., 2019), subdivided into five lithofacies units: massive and normally graduated gravels, cross stratified sands and gravelly sands, massive and laminated sands, clay silts and heterolithic facies (KUREČIĆ, 2017). Sandstones (lithoarenites) are limonitized and form thin interlayers within the sand The thickness of the Pliocene deposits is 200-400 m (PIKIJA, 1986).

Plio-Quaternary clastics (Pl, Q) unconformably cover various older deposits, from Jurassic to Pontian ages (Figure 2). They consist of sands, gravels and clays, rarely siltites, conglomerates, sandstones, and coal-clays and coal interlayers (ŠIKIĆ, 2014b; PIKIJA, 1986). Sands and gravels alternate irregularly forming interlayers and lens-shaped bodies. Clays are deposited as $\mathrm{cm}$ to $\mathrm{dm}$ thick interlayers and lens-shaped bodies within the sands. The thickness of these deposits ranges between 50 to $100 \mathrm{~m}$.

Loess (Pleistocene-1) is a clayey-sandy silt of aeolian origin and covers older, Neogene deposits (PIKIJA, 1986). The main mineral constituent is quartz (70\%), followed by feldspar $(16 \%)$, rock fragments $(10 \%)$ and muscovite $(4 \%)$, with a carbonate content of up to $13 \%$. Carbonate and limonite concretions are often observed. Around Petrinja and Cerje, lenticular interlayers of fine-grained gravels are also seen. Loess has characteristic vertical fractures forming loess bluffs. The thickness of loess deposits is up to $30 \mathrm{~m}$.

Holocene alluvial sediments (a, ap) are the most widespread Quaternary sediments in the area. Alluvial plain sediments are located in the Sava River valley on the Lekenička Poljana - Sisak - Hrastelnica line (PIKIJA, 1986). Silts predominate over sands and gravels. Oxbow lake sediments form characteristic arched and elongated depressions, filled with mud, silt and sand deposited during floods. These deposits are usually up to $5 \mathrm{~m}$ thick.

Holocene swamp deposits (b) are observed in the morphologically lowest parts of the Sava and Kupa River valleys. They are mostly composed of dark clays and clayey silts rich in organic matter (PIKIJA, 1986) 1 to 4 m thick.

Diluvial and proluvial sediments (dpr) are predominantly represented by sands and silts, although gravels and larger boulders can also be observed. They formed by surface water runoff 
and torrential flows from elevated areas, i.e. as a result of the combined seasonal deposition from mountain streams and creeks and ponds formed along margins of larger river valleys (e.g. the Sava, Una and Glina Rivers) (PIKIJA, 1986; ŠIKIĆ, 2014b). The thickness of these deposits is variable, locally it exceeds $10 \mathrm{~m}$.

\subsection{Tectogenesis of the research area}

The oldest exposed Jurassic and Cretaceous rock complexes of the Banija region are at least partly of ophiolitic origin, emplaced here as a result of obduction that began during the Middle Jurassic and terminated during Middle Cretaceous times (SCHMID et al., 2020) for a regional tectonic aspect. The Upper Cretaceous volcano-sedimentary complex is locally characterised by pelagic "Scaglia-type" limestones and turbidites, interlayered with pillow basalts and associated with a bimodal igneous succession comprising gabbros, doleritic dykes and rhyolites, all well exposed in the neighbouring Kozara Mts. in northern Bosnia and Herzegovina. There, this complex is interpreted as originating from a deep basin floored by oceanic lithosphere, that probably represented a remnant of the Vardar Ocean (USTASZEWSKI et al., 2009), which finally closed during the Palaeogene along the Sava suture zone (PAMIĆ, 2002; USTASZEWSKI et al., 2010; SCHMID et al., 2020). During the Early and Middle Miocene, the research area was affected by regional-scale extension linked to rift-related subsidence and formation of the Pannonian basin system, that in this area resulted in formation of the Sava basin and the neighbouring Karlovac basin (TOMLJENOVIĆ \& CSONTOS, 2001; SAFTIĆ et al., 2003; USTASZEWSKI et al., 2010). After the Pontian and during the Plio-Quaternary the extensional tectonic regime in the Pannonian basin was replaced by reginal-scale compression (BADA et al., 2007), which led to local inversion of previously active normal faults into faults with reverse sense of slip, often in association with newly formed reverse and strike slip faults (TOMLJENOVIĆ and CSONTOS, 2001; USTASZEWSKI et al., 2014), such as those responsible for the Zagreb (March 22, 2020) and the Petrinja (December 29, 2020) earthquakes, respectively.

\subsection{Seismicity}

Most of the recorded events of the Petrinja earthquake series (December 2020 - January 2021) so far are attributed to the Petrinja composite seismogenic source that is known in the European Database of Seismogenic Faults (BASILI et al., 2013) under the code HRCS027. Based on fault characteristics and seismological considerations, the maximum expected magnitude that is presumed for this source is 6.5 in the moment magnitude scale.

The strongest destructive $\mathrm{M}_{\mathrm{W}} 6.4$ earthquake occurred on the $29^{\text {th }}$ December 2020, 12:19 local time with an epicentre in the village of Strašnik, $6 \mathrm{~km} \mathrm{SW}$ of Petrinja at a preliminary estimated hypocentre depth of $10 \mathrm{~km}$. It caused the loss of seven lives and severe damage to buildings and infrastructure in the cities of Sisak, Petrinja, Glina, Dvor, Kravarsko and the surrounding villages, but also in the remote town of Zaprešić, some $60 \mathrm{~km} \mathrm{NW}$ of the epicentre. The intensity at the epicentre is estimated to be VIII-IX ${ }^{\circ}$ of European Macroseismic Scale (EMS), described as heavily damaging to destructive. Before this event there were three foreshocks, the strongest of which with a magnitude $5.2 \mathrm{M}_{\mathrm{w}}$ the day before the main shock. Currently, the seismic series is still ongoing with already more than 1000 earthquakes above magnitude $\mathrm{M}_{\mathrm{L}} 1.5$ recorded in the first couple of days after the mainshock. Aftershock seismicity is mostly located on the main seismogenic fault (HRCS027) with some earthquakes located on adjacent faults parallel to the main one. So far, recorded seismicity indicates that a $20 \mathrm{~km}$ long section of the fault ruptured with aftershock depths ranging from 5 to $15 \mathrm{~km}$.

Results from this up to January $13^{\text {th }} 2021$, suggest that in Sisak-Moslavina county, 33590 buildings were reported as damaged and 15626 were inspected by civil engineers and deemed as follows:

$-2292(15 \%)$ are classified as unusable;

- $1655(11 \%)$ are classified as temporarily unusable - detailed inspection needed;

- $1986(13 \%)$ are classified as temporarily unusable - emergency interventions needed;

$-5830(36 \%)$ are classified as usable with recommendations for action

$-410(3 \%)$ are classified as usable with no damage;

$-3453(22 \%)$ are classified as usable with no limitations.

Among these, more than 3000 building inspection reports encompass the problems related to the soil surrounding the building, and the necessity for detailed geotechnical and geological inspection.

According to the last census (2011) the population of SisakMoslavina county was 172 439, but in the most damaged areas (Petrinja, Sisak, Glina, Dvor and several smaller villages) the population was less than 90000 . Even if we ignore the emigration of the population in recent years, the number of damaged buildings in comparison with the population is substantial.

The direct damage of intensive ground-shaking on the buildings and infrastructure are evaluated by several services and numerous experts, mainly civil engineers. Besides, the same earthquake series also caused a variety of ground failures over a wider area, which represent additional, direct and indirect threats to the local community and their property, which have so far not been considered by those experts and Civil Protection services.

\section{THE PRELIMINARY INVENTORY}

The ground failure inventories related to particular seismic events or series are prepared to improve the ability to assess earthquaketriggered ground failure hazards. Such inventories enable the use of analysis of effects of a single triggering event on particular ground failures or effects. The inventories are most commonly related to particular countries or regions. Therefore, United States Geological Survey (USGS) created an openly accessible, centralized earthquake-triggered ground failure repository in the form of a ScienceBase Community to provide open access to data with the goal of accelerating research progress (SCHMITT, 2017). The digital inventories in that Community are created by both USGS and non-USGS authors.

Italy has a very extensive database on earthquake-induced ground effects, which has been upgraded over time. The database contains data regarding landslides, liquefaction, ground cracks, surface faulting and ground changes triggered by earthquakes of Mercalli epicentral intensity 8 or greater, that occurred in the last millennium (MARTINO et al., 2014). The same database was upgraded with new architecture in order to increase its usefulness for both Public Institutions and individual users (CAPRARI et al., 2018).

As already mentioned above, historical records of ground failures in Croatia are very scarce. Two catastrophic earthquakes with an estimated $\mathrm{X}^{\circ} \mathrm{MCS}$ scale are chronicled. For example, there is a legend about a possible earthquake that struck the island of Pag in the year 361 AD when, according to oral tradition, the 
city of Cissa collapsed into the sea. In 1667, the catastrophic Dubrovnik earthquake caused thousands of deaths (the estimations on number of victims differ), totally demolished almost the whole city, triggered rockfalls of huge blocks from Srđ mountain that caused great devastation and initiated a tsunami that damaged ships in the harbour (HERAK et al., 2017a).

More exact historical records about seismogenic ground effects are mainly related to the earthquake near Zagreb in 1880 . That earthquake with a magnitude of 6.3 initiated: liquefaction at several locations in the zone of Sava alluvium (Stupnik, Jarun, Resnik, Ivanja Reka, Drenje and Trstenik); raised the groundwater table (Krapinske Toplice, Novo Čiče, Lukač); created suffosion processes forming dolines (Stubičke Toplice); ground ruptures (Kašina) and triggered rockfalls (Severin na Kupi) (TORBAR, 1882).

In recent history, the earthquake near Zagreb $\mathrm{M}_{\mathrm{L}} 5.52020$ March $22^{\text {nd }} 2020$ caused the loss of one life and considerable material damage (ŠAVOR NOVAK et al., 2020), but ground failures were not reported. However, as reported by BAČIĆ et al. (2020), geological and geotechnical phenomena within the underlying soil yielded significant damage to some of the buildings constructed in the vicinity of identified faults and landslides.

The much stronger Petrinja $\mathrm{M}_{\mathrm{w}} 6.42020$ earthquake initiated various processes/effects, which can generally be divided into two main groups: ground failures and other ground effects (Table 1). Therefore, the database presented here is adjusted for up to date knowledge about historical and recent earthquake-triggered ground failures in Croatia. In this paper it presents data collected from $30^{\text {th }}$ of December 2020 until $15^{\text {th }}$ of January 2021 related to the Petrinja 2020-2021 earthquake series only, but as has already been stated, the database will be continuously updated online, at the HGI Web Map Service at www.hgi-cgs.hr.

It is important to point out that a single record in the database is for some processes/phenomena related to a single occurrence
Table 1. The earthquake-triggered ground effects and subdivisions included in the inventory.

\begin{tabular}{|c|c|c|c|}
\hline Ground effects & Processes & Phenomena/Feature & $\begin{array}{l}\text { No. of } \\
\text { occurrences / } \\
\text { locations }\end{array}$ \\
\hline \multirow{7}{*}{ Ground failures } & \multirow{2}{*}{ Subsidence dolines } & Dropout doline & 35 \\
\hline & & Suffosion doline & 4 \\
\hline & \multirow{3}{*}{ Liquefaction } & Sand boil & 85 \\
\hline & & Lateral spreading & 8 \\
\hline & & Subsidence & 6 \\
\hline & \multirow{2}{*}{ Landslides } & Slide & 36 \\
\hline & & Rockfall & 4 \\
\hline \multirow{3}{*}{$\begin{array}{l}\text { Other ground } \\
\text { effects }\end{array}$} & \multirow{2}{*}{ Geological } & Soil rupture & 20 \\
\hline & & Clouding of springwater & 1 \\
\hline & Infrastructural & Road rupture & 54 \\
\hline
\end{tabular}

of the phenomena, e.g. a subsidence doline or landslide. In other cases, single records in the database rather present locations or zones where multiple appearances of some process of phenomena are documented (i.e. sand boils, lateral spreading and different type of ruptures).

The inventory contains basic information about ground failures: No. (number of record), ID (label for the observation point), ground failure/effect type, coordinates (coordinate reference system-HTRS96 Croatia TM), data provider, processing date (field identification date, date of registration) and reliability (Table 2). The reliability of the data in the inventory is defined on the level of expertise by the reporter and the precision of spatial positioning. Therefore, the processes reported or verified by certified experts with exact coordinates are considered as highly confident. Unverified reports by non-professionals with approximate locations are considered to have low confidence. All cases in between are classified as medium confident.

Table 2. The preliminary inventory of ground failures and other ground effects after the Petrinja 2020 earthquake.

\begin{tabular}{|c|c|c|c|c|c|c|c|}
\hline No & ID & $\begin{array}{l}\text { Ground failure type } \\
\text { phenomena/process }\end{array}$ & $\mathrm{x}$ & $\mathrm{y}$ & Data provider & Processing date & $\begin{array}{l}\text { Data confidence } \\
\text { level }\end{array}$ \\
\hline & & SUBSIDENCE & & & & & \\
\hline 1 & IK1 & Dropout doline & 494638 & 5015706 & HGI & 13.01.2021. & high \\
\hline 2 & IK10 & Dropout doline & 494230 & 5016289 & HGI & 13.01.2021. & high \\
\hline 3 & IK11 & Dropout doline & 494219 & 5016193 & HGI & 13.01.2021. & high \\
\hline 4 & IK12 & Dropout doline & 494236 & 5016257 & HGI & 13.01.2021. & high \\
\hline 5 & IK13 & Dropout doline & 494010 & 5016789 & HGI & 13.01.2021. & high \\
\hline 6 & IK14 & Dropout doline & 494002 & 5016794 & HGI & 13.01.2021. & high \\
\hline 7 & IK17 & Dropout doline & 493781 & 5017077 & HGI & 13.01.2021. & high \\
\hline 8 & IK18 & Dropout doline & 493677 & 5017093 & HGI & 13.01.2021. & high \\
\hline 9 & IK19 & Dropout doline & 493597 & 5017146 & HGI & 13.01.2021. & high \\
\hline 10 & IK2 & Dropout doline & 494663 & 5015641 & HGI & 13.01.2021. & high \\
\hline 11 & IK20 & Dropout doline & 493589 & 5017180 & HGI & 13.01.2021. & high \\
\hline 12 & IK21 & Dropout doline & 493598 & 5017171 & HGI & 13.01.2021. & high \\
\hline 13 & IK22 & Dropout doline & 493425 & 5017321 & HGI & 13.01.2021. & high \\
\hline 14 & IK23 & Dropout doline & 493431 & 5017341 & HGI & 13.01.2021. & high \\
\hline 15 & IK25 & Dropout doline & 493449 & 5017139 & HGI & 13.01.2021. & high \\
\hline 16 & IK26 & Dropout doline & 493459 & 5017136 & HGI & 13.01.2021. & high \\
\hline 17 & IK27 & Dropout doline & 493499 & 5017123 & HGI & 13.01.2021. & high \\
\hline 18 & IK28 & Dropout doline & 493556 & 5017112 & HGI & 13.01.2021. & high \\
\hline 19 & IK29 & Dropout doline & 493564 & 5017102 & HGI & 13.01.2021. & high \\
\hline 20 & IK30 & Dropout doline & 494514 & 5015761 & HGI & 13.01.2021. & high \\
\hline 21 & IK31 & Dropout doline & 494496 & 5015874 & HGI & 13.01.2021. & high \\
\hline
\end{tabular}




\begin{tabular}{|c|c|c|c|c|c|c|c|}
\hline No & ID & $\begin{array}{l}\text { Ground failure type } \\
\text { phenomena/process }\end{array}$ & $\mathrm{x}$ & $\mathrm{y}$ & Data provider & Processing date & $\begin{array}{l}\text { Data confidence } \\
\text { level }\end{array}$ \\
\hline 22 & IK32 & Dropout doline & 494462 & 5015878 & HGI & 13.01.2021. & high \\
\hline 23 & IK33 & Dropout doline & 494386 & 5015780 & HGI & 13.01.2021. & high \\
\hline 24 & IK34 & Dropout doline & 494321 & 5015781 & HGI & 13.01.2021. & high \\
\hline 25 & IK35 & Dropout doline & 493266 & 5016975 & HGI & 13.01.2021. & high \\
\hline 26 & IK4 & Dropout doline & 494186 & 5015950 & HGI & 13.01.2021. & high \\
\hline 27 & IK5 & Dropout doline & 494138 & 5015846 & HGI & 13.01.2021. & high \\
\hline 28 & IK6 & Dropout doline & 494100 & 5015895 & HGI & 13.01.2021. & high \\
\hline 29 & IK7 & Dropout doline & 494123 & 5016224 & HGI & 13.01.2021. & high \\
\hline 30 & IK8 & Dropout doline & 494120 & 5016287 & HGI & 13.01.2021. & high \\
\hline 31 & IK9 & Dropout doline & 494219 & 5016329 & HGI & 13.01.2021. & high \\
\hline 32 & IK-37 & Dropout doline & 493938 & 5016123 & HGI & 20.01.2021. & high \\
\hline 33 & IK-39 & Dropout doline & 493885 & 5016101 & HGI & 20.01.2021. & high \\
\hline 34 & IK-40 & Dropout doline & 494125 & 5016227 & HGI & 20.01.2021. & high \\
\hline 35 & IK-41 & Dropout doline & 492843 & 5017598 & HGI & 20.01.2021. & high \\
\hline 36 & IK15 & Suffosion doline & 493911 & 5016862 & HGI & 13.01.2021. & high \\
\hline 37 & IK16 & Suffosion doline & 493861 & 5017003 & HGI & 13.01.2021. & high \\
\hline 38 & IK24 & Suffosion doline & 493613 & 5016984 & HGI & 13.01.2021. & high \\
\hline \multirow[t]{2}{*}{39} & IK3 & Suffosion doline & 494656 & 5015991 & HGI & 13.01.2021. & high \\
\hline & & LIQUEFACTION & & & & & \\
\hline 40 & P-NDTV-12b & Sand boil & 481448 & 5032639 & HGI & 05.01 .2021 & high \\
\hline 41 & P-NDTV-13a & Sand boil & 481504 & 5032582 & HGI & 05.01 .2021$. & high \\
\hline 42 & PE-NDTV-02 & Sand boil & 481883 & 5032780 & HGI & 05.01.2021. & high \\
\hline 43 & PE-NDTV-03 & Sand boil & 481838 & 5032783 & HGI & 05.01.2021. & high \\
\hline 44 & PE-NDTV-04 & Sand boil & 481834 & 5032784 & HGI & 05.01.2021. & high \\
\hline 45 & PE-NDTV-05 & Sand boil & 481814 & 5032793 & HGI & 05.01.2021. & high \\
\hline 46 & PE-NDTV-06 & Sand boil & 481827 & 5032750 & HGI & 05.01.2021. & high \\
\hline 47 & PE-NDTV-07 & Sand boil & 481800 & 5032746 & HGI & 05.01.2021. & high \\
\hline 48 & PE-NDTV-08 & Sand boil & 481736 & 5032716 & HGI & 05.01.2021. & high \\
\hline 49 & PE-NDTV-09 & Sand boil & 481653 & 5032627 & HGI & 05.01.2021. & high \\
\hline 50 & PE-NDTV-10 & Sand boil & 481629 & 5032610 & HGI & 05.01.2021. & high \\
\hline 51 & PE-NDTV-11 & Sand boil & 481436 & 5032602 & HGI & 05.01.2021. & high \\
\hline 52 & PE-NDTV-15 & Sand boil & 481837 & 5032832 & HGI & 05.01.2021. & high \\
\hline 53 & PE-NDTV-16 & Sand boil & 481783 & 5032842 & HGI & 05.01.2021. & high \\
\hline 54 & $\mathrm{Si}-01$ & Sand boil & 491454 & 5037182 & HGI & 05.01.2021. & high \\
\hline 55 & $\mathrm{Si}-04$ & Sand boil & 491773 & 5036963 & HGI & 05.01.2021. & high \\
\hline 56 & $\mathrm{Si}-05$ & Sand boil & 491670 & 5037051 & HGI & 05.01.2021. & high \\
\hline 57 & V-01 & Sand boil & 481401 & 5033976 & HGI & 09.01.2021. & high \\
\hline 58 & RMT-11 & Sand boil & 476846 & 5040083 & HGI & 04.01.2021. & high \\
\hline 59 & RMT-12 & Sand boil & 476693 & 5040607 & HGI & 04.01.2021. & high \\
\hline 60 & RMT-17 & Sand boil & 470568 & 5030270 & HGI & 04.01.2021. & high \\
\hline 61 & RMT-19 & Sand boil & 472346 & 5038019 & HGI & 05.01.2021. & high \\
\hline 62 & RMT-20 & Sand boil & 468309 & 5036590 & HGI & 05.01 .2021$. & high \\
\hline 63 & RMT-21 & Sand boil & 468320 & 5036356 & HGI & 05.01.2021. & high \\
\hline 64 & RMT-22 & Sand boil & 468391 & 5036401 & HGI & 05.01.2021. & high \\
\hline 65 & RMT-23 & Sand boil & 468423 & 5036435 & HGI & 05.01.2021. & high \\
\hline 66 & RMT-28a & Sand boil & 469995 & 5025866 & HGI & 05.01.2021. & high \\
\hline 67 & RMT-34b & Sand boil & 477383 & 5041120 & HGI & 07.01.2021. & high \\
\hline 68 & RMT-35a & Sand boil & 477582 & 5040954 & HGI & 07.01.2021. & high \\
\hline 69 & RMT-36 & Sand boil & 477608 & 5040971 & HGI & 07.01.2021. & high \\
\hline 70 & RMT-37 & Sand boil & 477681 & 5040990 & HGI & 07.01.2021. & high \\
\hline 71 & RMT-37a & Sand boil & 477696 & 5041003 & HGI & 07.01.2021. & high \\
\hline 72 & RMT-37c & Sand boil & 477722 & 5041036 & HGI & 07.01.2021. & high \\
\hline 73 & RMT-49 & Sand boil & 470030 & 5033099 & HGI & 07.01.2021. & medium \\
\hline 74 & RMT-50 & Sand boil & 465677 & 5046800 & HGI & 07.01.2021. & high \\
\hline 75 & MV-1 & Sand boil & 481233 & 5034723 & HGI & 30.12 .2020 . & high \\
\hline 76 & MV-26 & Sand boil & 481241 & 5034479 & HGI & 04.01.2021. & high \\
\hline 77 & MV-28 & Sand boil & 485589 & 5036755 & HGI & 04.01.2021. & high \\
\hline 78 & MV-29 & Sand boil & 487149 & 5035782 & HGI & 04.01.2021. & high \\
\hline 79 & MV-31 & Sand boil & 481356 & 5033058 & HGI & 05.01.2021. & high \\
\hline
\end{tabular}




\begin{tabular}{|c|c|c|c|c|c|c|c|}
\hline No & ID & $\begin{array}{l}\text { Ground failure type } \\
\text { phenomena/process }\end{array}$ & $\mathrm{x}$ & $\mathrm{y}$ & Data provider & Processing date & $\begin{array}{l}\text { Data confidence } \\
\text { level }\end{array}$ \\
\hline 80 & MV-32 & Sand boil & 481392 & 5032955 & HGI & 05.01.2021. & high \\
\hline 81 & MV-34 & Sand boil & 493935 & 5042867 & HGI & 05.01.2021. & high \\
\hline 82 & MV-35 & Sand boil & 493257 & 5042439 & HGI & 05.01 .2021 & high \\
\hline 83 & MV-36 & Sand boil & 492637 & 5042213 & HGI & 05.01 .2021 & high \\
\hline 84 & MV-37 & Sand boil & 492202 & 5042157 & HGI & 05.01.2021. & high \\
\hline 85 & NP-13 & Sand boil & 487317 & 5035347 & HGI & 04.01.2021. & high \\
\hline 86 & NP-15 & Sand boil & 479994 & 5033591 & HGI & 05.01 .2021 & high \\
\hline 87 & NP-22 & Sand boil & 492859 & 5041123 & HGI & 07.01 .2021$. & high \\
\hline 88 & NP-23 & Sand boil & 492724 & 5040426 & HGI & 07.01.2021. & high \\
\hline 89 & NP-26 & Sand boil & 491978 & 5036711 & HGI & 07.01.2021. & high \\
\hline 90 & NP-30 & Sand boil & 471583 & 5028895 & HGI & 07.01.2021. & high \\
\hline 91 & NP-31 & Sand boil & 480133 & 5034511 & HGI & 13.01.2021. & high \\
\hline 92 & JB-1 & Sand boil & 468823 & 5025602 & HGI & 07.01 .2021$. & high \\
\hline 93 & JB-2 & Sand boil & 468416 & 5024971 & HGI & 07.01.2021. & high \\
\hline 94 & JB-6 & Sand boil & 468364 & 5022176 & HGI & 07.01 .2021$. & high \\
\hline 95 & JB-7 & Sand boil & 468298 & 5022237 & HGI & 07.01.2021. & high \\
\hline 96 & ŠP-7 & Sand boil & 485645 & 5035151 & HGI & 04.01 .2021$. & high \\
\hline 97 & ŠP-8 & Sand boil & 491626 & 5042295 & HGI & 05.01 .2021 & high \\
\hline 98 & ŠP-9 & Sand boil & 491659 & 5042255 & HGI & 05.01.2021. & high \\
\hline 99 & ŠP-17 & Sand boil & 485075 & 5035494 & HGI & 05.01 .2021$. & high \\
\hline 100 & TSS-2 & Sand boil & 481298 & 5034640 & RGNf & 05.01.2021. & high \\
\hline 101 & TSS-3 & Sand boil & 493197 & 5042455 & RGNf & 05.01 .2021 & high \\
\hline 102 & GEOT-4 & Sand boil & 481485 & 5032868 & GEOT & 12.01.2021. & medium \\
\hline 103 & GEOT-12 & Sand boil & 481786 & 5035259 & GEOT & 12.01.2021. & medium \\
\hline 104 & GEOT-14 & Sand boil & 490382 & 5039444 & GEOT & 12.01.2021. & medium \\
\hline 105 & GEOT-28 & Sand boil & 481755 & 5033624 & GEOT & 12.01.2021. & medium \\
\hline 106 & GEOT-29 & Sand boil & 490464 & 5039161 & GEOT & 12.01.2021. & medium \\
\hline 107 & GEOT-30 & Sand boil & 467978 & 5022537 & GEOT & 12.01.2021. & medium \\
\hline 108 & GEOT-31 & Sand boil & 481632 & 5032863 & GEOT & 12.01.2021. & medium \\
\hline 109 & GEOT-32 & Sand boil & 481389 & 5032981 & GEOT & 12.01.2021. & medium \\
\hline 110 & NVI-05 & Sand boil & 481477 & 5032892 & HGI & 19.01.2021. & high \\
\hline 111 & NVI-05a & Sand boil & 481463 & 5032854 & HGI & 19.01.2021. & high \\
\hline 112 & NVI-06 & Sand boil & 481505 & 5032792 & HGI & 19.01 .2021 & high \\
\hline 113 & NVI-07 & Sand boil & 481507 & 5032791 & HGI & 19.01.2021. & high \\
\hline 114 & NVI-10 & Sand boil & 481372 & 5032989 & HGI & 19.01.2021. & high \\
\hline 115 & GTI-01 & Sand boil & 481114 & 5034850 & GTI & 20.01.2021. & high \\
\hline 116 & GTI-02a & Sand boil & 481882 & 5032867 & GTI & 20.01.2021. & high \\
\hline 117 & GTI-02b & Sand boil & 481878 & 5032825 & GTI & 20.01 .2021$. & high \\
\hline 118 & VI-03 & Sand boil & 476421 & 5040754 & HGI & 20.01 .2021$. & high \\
\hline 119 & VI-09 & Sand boil & 475259 & 5037960 & HGI & 20.01 .2021$. & high \\
\hline 120 & VI-10 & Sand boil & 475561 & 5038219 & HGI & 20.01 .2021$. & high \\
\hline 121 & VI-11 & Sand boil & 475469 & 5038256 & HGI & 20.01 .2021$. & high \\
\hline 122 & VI-12 & Sand boil & 475035 & 5037857 & HGI & 20.01.2021. & high \\
\hline 123 & VI-13 & Sand boil & 475055 & 5037862 & HGI & 20.01 .2021$. & high \\
\hline 124 & VI-15 & Sand boil & 473643 & 5038416 & HGI & 20.01 .2021$. & high \\
\hline 131 & $\mathrm{Si}-02$ & Lateral spreading & 491660 & 5036857 & HGI & 05.01 .2021 & high \\
\hline 132 & RMT-35 & Lateral spreading & 477562 & 5040939 & HGI & 07.01 .2021$. & high \\
\hline 133 & NP-12 & Lateral spreading & 487151 & 5035769 & HGI & 04.01 .2021 & high \\
\hline 134 & NP-21 & Lateral spreading & 492335 & 5042079 & HGI & 05.01 .2021$. & high \\
\hline 135 & GTI-06 & Lateral spreading & 481089 & 5034247 & GTI & 20.01.2021. & high \\
\hline 136 & VI-06 & Lateral spreading & 475295 & 5037837 & HGI & 20.01 .2021$. & high \\
\hline 137 & VI-07 & Lateral spreading & 475218 & 5037965 & HGI & 20.01.2021. & high \\
\hline 138 & VI-08 & Lateral spreading & 475348 & 5037831 & HGI & 20.01 .2021$. & high \\
\hline 125 & P-NDTV-05a & Subsidence & 481812 & 5032798 & HGI & 05.01 .2021$. & high \\
\hline 126 & P-NDTV-06a & Subsidence & 481825 & 5032746 & HGI & 05.01 .2021$. & high \\
\hline 127 & P-NDTV-11a & Subsidence & 481438 & 5032609 & HGI & 05.01 .2021$. & high \\
\hline 128 & P-NDTV-12a & Subsidence & 481471 & 5032626 & HGI & 05.01.2021. & high \\
\hline 129 & $\mathrm{Si}-03$ & Subsidence & 491818 & 5036749 & HGI & 05.01 .2021$. & high \\
\hline 130 & MV-37a & Subsidence & 492202 & 5042157 & HGI & 05.01.2021. & high \\
\hline
\end{tabular}




\begin{tabular}{|c|c|c|c|c|c|c|c|}
\hline No & ID & $\begin{array}{l}\text { Ground failure type } \\
\text { phenomena/process }\end{array}$ & $\mathrm{x}$ & $\mathrm{y}$ & Data provider & Processing date & $\begin{array}{l}\text { Data confidence } \\
\text { level }\end{array}$ \\
\hline & & LANDSLIDES & & & & & \\
\hline 139 & PE-NDTV-01 & Slide & 482878 & 5031095 & HGI & 05.01.2021. & high \\
\hline 140 & PE-NDTV-12 & Slide & 481474 & 5032619 & HGI & 05.01.2021. & high \\
\hline 141 & PE-NDTV-13 & Slide & 481496 & 5032594 & HGI & 05.01.2021. & high \\
\hline 142 & PE-NDTV-14 & Slide & 481511 & 5032676 & HGI & 05.01.2021. & high \\
\hline 143 & NP-16 & Slide & 482924 & 5025926 & HGI & 05.01.2021. & high \\
\hline 144 & ŠP-1 & Slide & 480887 & 5030303 & HGI & 04.01.2021. & high \\
\hline 145 & S̆P-3 & Slide & 479527 & 5029032 & HGI & 04.01.2021. & high \\
\hline 146 & TSS-9 & Slide & 464747 & 5024336 & RGNf & 05.01 .2021$. & high \\
\hline 147 & GEOT-1 & Slide & 464873 & 5027706 & GEOT & 12.01.2021. & medium \\
\hline 148 & GEOT-2 & Slide & 464471 & 5043053 & GEOT & 12.01.2021. & medium \\
\hline 149 & GEOT-3 & Slide & 475922 & 5031004 & GEOT & 12.01.2021. & medium \\
\hline 150 & GEOT-6 & Slide & 500430 & 5010142 & GEOT & 12.01.2021. & medium \\
\hline 151 & GEOT-7 & Slide & 468013 & 5022625 & GEOT & 12.01.2021. & medium \\
\hline 152 & GEOT-8 & Slide & 485421 & 5022788 & GEOT & 12.01.2021. & medium \\
\hline 153 & GEOT-10 & Slide & 482610 & 5023631 & GEOT & 12.01.2021. & low \\
\hline 154 & GEOT-11 & Slide & 467962 & 5022471 & GEOT & 12.01.2021. & low \\
\hline 155 & GEOT-13 & Slide & 480890 & 5032753 & GEOT & 12.01.2021. & medium \\
\hline 156 & GEOT-17 & Slide & 476987 & 5032775 & GEOT & 12.01.2021. & low \\
\hline 157 & GEOT-18 & Slide & 483558 & 5028393 & GEOT & 12.01.2021. & medium \\
\hline 158 & GEOT-21 & Slide & 480961 & 5034683 & GEOT & 12.01.2021. & medium \\
\hline 159 & GEOT-22 & Slide & 484231 & 5027591 & GEOT & 12.01.2021. & medium \\
\hline 160 & GEOT-25 & Slide & 482973 & 5026073 & GEOT & 12.01.2021. & medium \\
\hline 161 & GEOT-27 & Slide & 486499 & 5017777 & GEOT & 12.01.2021. & medium \\
\hline 162 & IK-KLZ1 & Slide & 490843 & 5018292 & HGI & 13.01.2021. & medium \\
\hline 163 & NVI-01 & Slide & 506036 & 5020302 & HGI & 19.01.2021. & high \\
\hline 164 & NVI-02 & Slide & 495761 & 5028047 & HGI & 19.01.2021. & high \\
\hline 165 & NVI-08 & Slide & 481404 & 5032745 & HGI & 19.01.2021. & high \\
\hline 166 & GTI-03a & Slide & 470225 & 5026717 & GTI & 20.01.2021. & high \\
\hline 167 & GTI-03b & Slide & 470238 & 5026682 & GTI & 20.01.2021. & high \\
\hline 168 & GTI-03c & Slide & 470121 & 5026487 & GTI & 20.01.2021. & high \\
\hline 169 & VI-01 & Slide & 477421 & 5040997 & HGI & 20.01.2021. & high \\
\hline 170 & VI-02 & Slide & 477694 & 5040845 & HGI & 20.01.2021. & high \\
\hline 171 & VI-04 & Slide & 473997 & 5038388 & HGI & 20.01.2021. & high \\
\hline 172 & VI-05 & Slide & 475133 & 5037725 & HGI & 20.01 .2021$. & high \\
\hline 173 & VI-17 & Slide & 472210 & 5038568 & HGI & 20.01.2021. & high \\
\hline 174 & VI-18 & Slide & 472124 & 5038608 & HGI & 20.01.2021. & high \\
\hline 175 & HR-DN-02 & Rockfall & 482859 & 5028347 & HGI & 05.01.2021. & high \\
\hline 176 & RMT-24 & Rockfall & 462393 & 5031336 & HGI & 05.01.2021. & high \\
\hline 177 & ŠP-16 & Rockfall & 483128 & 5028418 & HGI & 04.01.2021. & high \\
\hline \multirow[t]{2}{*}{178} & IK-ODR & Rockfall & 480287 & 5027202 & HGI & 14.01.2021. & high \\
\hline & & GEOLOGICAL & & & & & \\
\hline 179 & RMT-5 & Soil rupture & 475149 & 5033810 & HGI & 04.01.2021. & high \\
\hline 180 & RMT-7 & Soil rupture & 474978 & 5032745 & HGI & 04.01.2021. & high \\
\hline 181 & RMT-8 & Soil rupture & 474935 & 5032603 & HGI & 04.01.2021. & high \\
\hline 182 & RMT-10 & Soil rupture & 474902 & 5032794 & HGI & 04.01.2021. & high \\
\hline 183 & RMT-14 & Soil rupture & 474043 & 5038129 & HGI & 04.01.2021. & high \\
\hline 184 & RMT-27 & Soil rupture & 471742 & 5027712 & HGI & 05.01.2021. & high \\
\hline 185 & RMT-34a & Soil rupture & 477383 & 5041120 & HGI & 07.01 .2021 & high \\
\hline 186 & RMT-37b & Soil rupture & 477704 & 5041022 & HGI & 07.01.2021. & high \\
\hline 187 & RMT-38 & Soil rupture & 477764 & 5041061 & HGI & 07.01.2021. & high \\
\hline 188 & MV-11 & Soil rupture & 478416 & 5031477 & HGI & 30.12 .2020 & high \\
\hline 189 & MV-20 & Soil rupture & 478261 & 5031617 & HGI & 04.01 .2021$. & high \\
\hline 190 & NP-25 & Soil rupture & 491818 & 5036749 & HGI & 07.01 .2021 & high \\
\hline 191 & TSS-4 & Soil rupture & 492419 & 5042102 & RGNf & 05.01.2021. & high \\
\hline 192 & TSS-6 & Soil rupture & 465989 & 5044352 & RGNf & 05.01.2021. & high \\
\hline 193 & TSS- 8 & Soil rupture & 465701 & 5046760 & RGNf & 05.01.2021. & high \\
\hline 194 & GEOT-9 & Soil rupture & 475062 & 5032742 & GEOT & 12.01.2021. & medium \\
\hline 195 & GEOT-16 & Soil rupture & 479668 & 5039767 & GEOT & 12.01.2021. & medium \\
\hline
\end{tabular}




\begin{tabular}{|c|c|c|c|c|c|c|c|}
\hline No & ID & $\begin{array}{l}\text { Ground failure type } \\
\text { phenomena/process }\end{array}$ & $\mathrm{x}$ & $\mathrm{y}$ & Data provider & Processing date & $\begin{array}{l}\text { Data confidence } \\
\text { level }\end{array}$ \\
\hline 196 & GEOT-19 & Soil rupture & 481759 & 5035271 & GEOT & 12.01.2021. & medium \\
\hline 197 & VI-14 & Soil rupture & 473739 & 5038352 & HGI & 20.01.2021. & high \\
\hline 198 & VI-16 & Soil rupture & 472650 & 5038286 & HGI & 20.01.2021. & high \\
\hline \multirow[t]{2}{*}{253} & HR-DN-01 & Clouding of springwater & 482977 & 5028375 & HGI & 05.01.2021. & high \\
\hline & & INFRASTRUCTURAL & & & & & \\
\hline 199 & $\mathrm{Si}-06$ & Road rupture & 490317 & 5039630 & HGI & 05.01.2021. & high \\
\hline 200 & RMT-3 & Road rupture & 477178 & 5033155 & HGI & 04.01.2021. & high \\
\hline 201 & RMT-13 & Road rupture & 476121 & 5036980 & HGI & 04.01.2021. & high \\
\hline 202 & RMT-15 & Road rupture & 479011 & 5032783 & HGI & 04.01.2021. & high \\
\hline 203 & RMT-16 & Road rupture & 470485 & 5030089 & HGI & 04.01.2021. & high \\
\hline 204 & RMT-18 & Road rupture & 473752 & 5036859 & HGI & 05.01.2021. & high \\
\hline 205 & RMT-25 & Road rupture & 471772 & 5027624 & HGI & 05.01.2021. & high \\
\hline 206 & RMT-26 & Road rupture & 471744 & 5027688 & HGI & 05.01.2021. & high \\
\hline 207 & RMT-28 & Road rupture & 469995 & 5025866 & HGI & 05.01.2021. & high \\
\hline 208 & RMT-31 & Road rupture & 479609 & 5041418 & HGI & 07.01.2021. & high \\
\hline 209 & RMT-32 & Road rupture & 479117 & 5041335 & HGI & 07.01.2021. & high \\
\hline 210 & RMT-33 & Road rupture & 477440 & 5041161 & HGI & 07.01.2021. & high \\
\hline 211 & RMT-34 & Road rupture & 477383 & 5041120 & HGI & 07.01.2021. & high \\
\hline 212 & RMT-40 & Road rupture & 470620 & 5039412 & HGI & 07.01.2021. & high \\
\hline 213 & RMT-41 & Road rupture & 469622 & 5038340 & HGI & 07.01.2021. & high \\
\hline 214 & RMT-42 & Road rupture & 469564 & 5038235 & HGI & 07.01.2021. & high \\
\hline 215 & RMT-43 & Road rupture & 469564 & 5038202 & HGI & 07.01.2021. & high \\
\hline 216 & RMT-44 & Road rupture & 469530 & 5038129 & HGI & 07.01.2021. & high \\
\hline 217 & RMT-45 & Road rupture & 469402 & 5038127 & HGI & 07.01.2021. & high \\
\hline 218 & RMT-46 & Road rupture & 469364 & 5038134 & HGI & 07.01.2021. & high \\
\hline 219 & RMT-47 & Road rupture & 469213 & 5038807 & HGI & 07.01.2021. & medium \\
\hline 220 & RMT-48 & Road rupture & 468228 & 5037625 & HGI & 07.01.2021. & high \\
\hline 221 & RMT-51 & Road rupture & 464773 & 5042397 & HGI & 07.01.2021. & high \\
\hline 222 & RMT-52 & Road rupture & 465548 & 5043364 & HGI & 07.01.2021. & high \\
\hline 223 & RMT-53 & Road rupture & 466068 & 5044321 & HGI & 07.01.2021. & high \\
\hline 224 & MV-2 & Road rupture & 481193 & 5034203 & HGI & 30.12 .2020 & high \\
\hline 225 & MV-3 & Road rupture & 478036 & 5032639 & HGI & 30.12 .2020 . & high \\
\hline 226 & MV-8 & Road rupture & 479017 & 5032643 & HGI & 30.12 .2020$. & medium \\
\hline 227 & MV-9 & Road rupture & 478292 & 5031591 & HGI & 30.12 .2020 . & high \\
\hline 228 & MV-10 & Road rupture & 478342 & 5031565 & HGI & 30.12 .2020 . & high \\
\hline 229 & MV-12 & Road rupture & 478544 & 5031382 & HGI & 30.12 .2020 & high \\
\hline 230 & MV-13 & Road rupture & 478842 & 5031213 & HGI & 30.12 .2020 . & high \\
\hline 231 & MV-14 & Road rupture & 479186 & 5031158 & HGI & 30.12 .2020 . & high \\
\hline 232 & MV-15 & Road rupture & 479385 & 5031222 & HGI & 30.12 .2020 & high \\
\hline 233 & MV-16 & Road rupture & 479975 & 5031360 & HGI & 30.12 .2020 . & high \\
\hline 234 & MV-17 & Road rupture & 480701 & 5030415 & HGI & 30.12 .2020 & high \\
\hline 235 & MV-18 & Road rupture & 480379 & 5030066 & HGI & 30.12 .2020 . & high \\
\hline 236 & MV-19 & Road rupture & 480192 & 5029947 & HGI & 30.12 .2020 . & medium \\
\hline 237 & MV-21 & Road rupture & 478159 & 5031553 & HGI & 04.01 .2021$. & high \\
\hline 238 & MV-22 & Road rupture & 478950 & 5032157 & HGI & 04.01.2021. & high \\
\hline 239 & MV-24 & Road rupture & 481153 & 5033888 & HGI & 04.01.2021. & high \\
\hline 240 & MV-25 & Road rupture & 481370 & 5034062 & HGI & 04.01.2021. & high \\
\hline 241 & MV-27 & Road rupture & 481360 & 5034587 & HGI & 04.01.2021. & high \\
\hline 242 & MV-30 & Road rupture & 481419 & 5033078 & HGI & 04.01.2021. & high \\
\hline 243 & MV-33 & Road rupture & 483311 & 5026330 & HGI & 05.01 .2021$. & high \\
\hline 244 & JB-3 & Road rupture & 470110 & 5025591 & HGI & 07.01.2021. & high \\
\hline 245 & ŠP-5 & Road rupture & 483356 & 5026406 & HGI & 04.01.2021. & high \\
\hline 246 & ŠP-10 & Road rupture & 485695 & 5035094 & HGI & 05.01 .2021$. & high \\
\hline 247 & ŠP-11 & Road rupture & 480333 & 5027108 & HGI & 04.01.2021. & high \\
\hline 248 & ŠP-12 & Road rupture & 479690 & 5026544 & HGI & 04.01.2021. & high \\
\hline 249 & ŠP-14 & Road rupture & 480866 & 5030242 & HGI & 04.01.2021. & high \\
\hline 250 & ŠP-18 & Road rupture & 487411 & 5025085 & HGI & 05.01.2021. & high \\
\hline 251 & TSS-7 & Road rupture & 478145 & 5031572 & RGNf & 05.01.2021. & high \\
\hline 252 & GTI-07 & Road rupture & 485160 & 5034297 & GTI & 20.01.2021. & high \\
\hline
\end{tabular}


a)

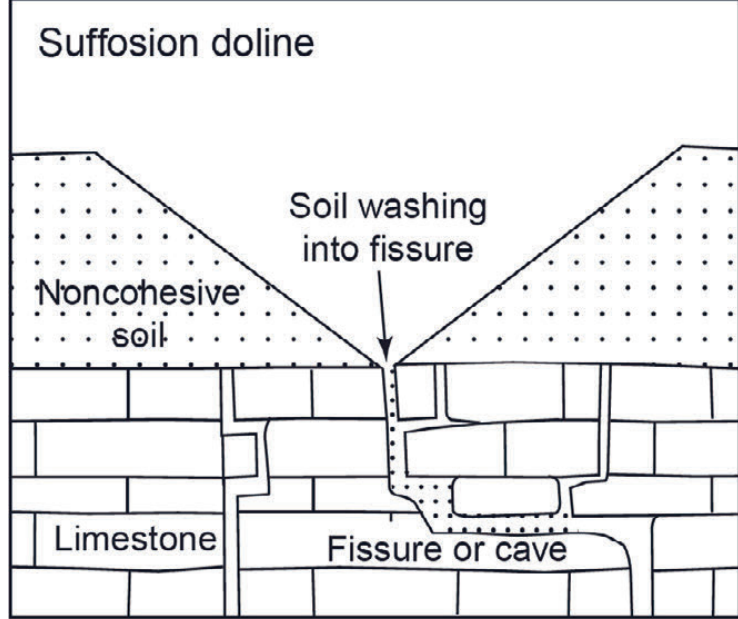

b)

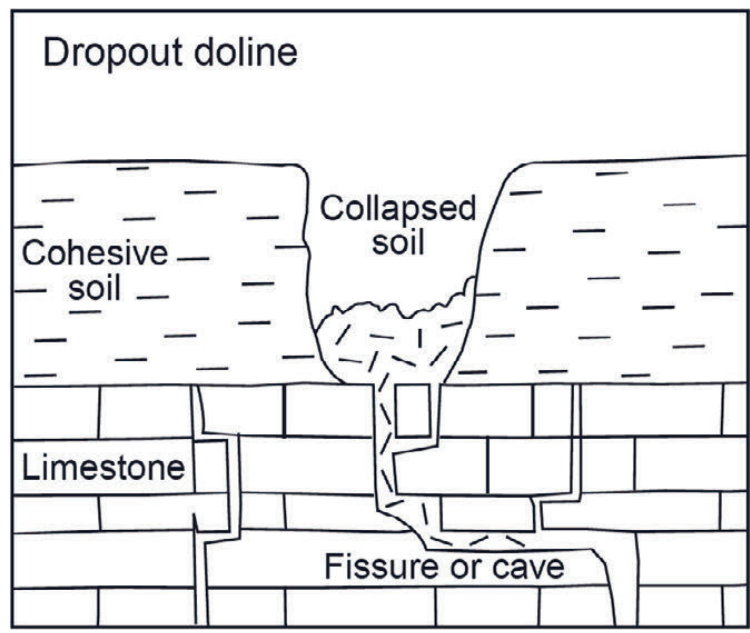

Figure 3. The sketches of: a) suffosion doline and b) dropout doline. Reproduced from WALTHAM \& FOOKES (2003).

\subsection{Ground failures}

Ground failures are largely related to subsidence, liquefaction, and landsliding processes.

\subsubsection{Subsidence dolines}

Subsidence is the sudden sinking or gradual downward settling of the Earth's surface with little or no horizontal motion (BATES \& JACKSON, 1980). Subsidence may be caused by different natural geological processes, but here subsidence caused by suffosion or liquefaction is of primary concern.

The ground shaking caused by the Petrinja earthquake series triggered rapid development of subsidence dolines in the wider Mečenčani area, about $20 \mathrm{~km}$ SE of the mainshock epicentre. Actually, in this area dolines were registered throughout history and obviously, strong earthquake accelerated the process significantly. Theoretically, two types of subsidence dolines are recognised: suffosion and dropout dolines (KRANJC, 2013; Figure 3). Both types are developed by suffosion, a filtration process in which fine particles are washed away from cover sediments overlying carbonate bedrock or other soluble rocks, into their karstified fissures or caverns. In the area of Mečenčani, mainly dropout dolines are developed. In this type of doline cover sediments are evacuated downwards through solution pipes in the bedrock creating a void at the bedrock-sediment contact that enlarges by upwards propagation through the clastic coverbeds (WILLIAMS, 2004; Figure 3).

At the time of writing this manuscript, 35 records were entered in the inventory (Figure 4, Table 1), but up to February $15^{\text {th }}$ 2021, more than ninety (90) subsidence dolines were registered in the area between Borojevići and Mečenčani in the Donji Kukuruzari municipality. The great majority of these are classified as dropout dolines formed by the sudden (or over a few hours) collapse of cover sediments into the underground voids, forming steep-sided cylindrical depressions. The majority of the dolines are between 1 and $10 \mathrm{~m}$ in diameter, but the largest one is more
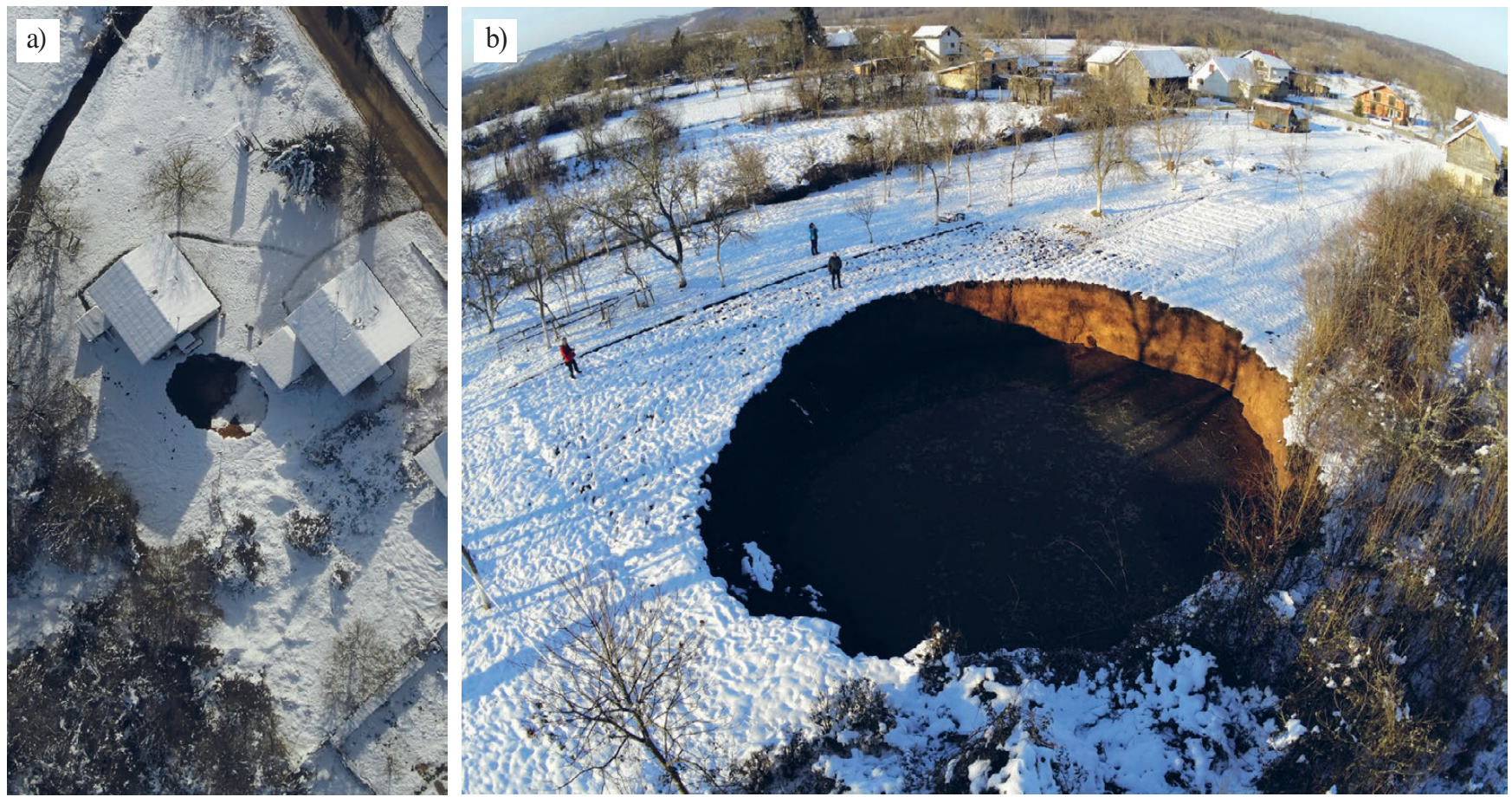

Figure 4. Dropout dolines in Mečenčani village: a) endangering adjacent houses (observation point IK31), b) the biggest doline, more than 20m in diameter and about $12 \mathrm{~m}$ deep (observation point IK4). 

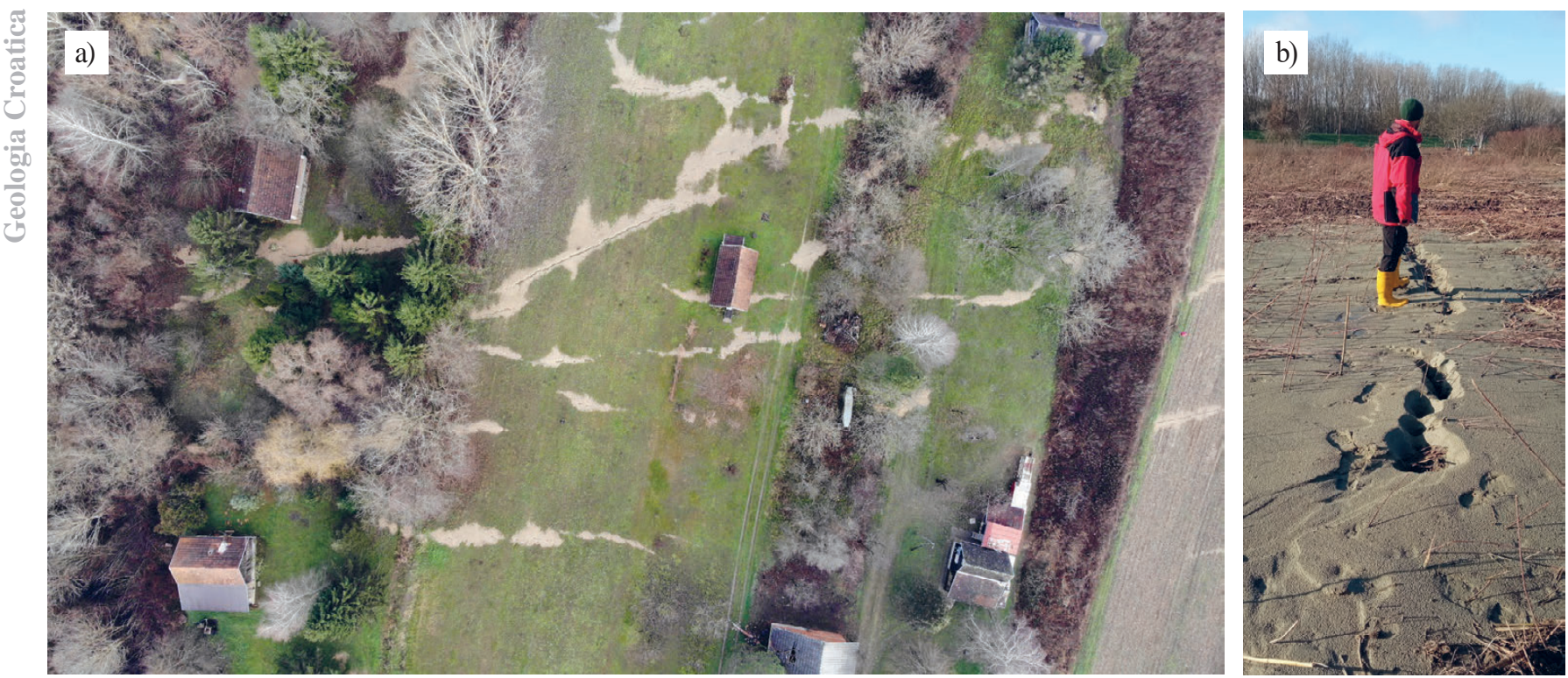

Figure 5. The sand boiling features along linear fractures: a) in Letovanić (aerial photo); b) in Sisak (observation point Si-04).

than $20 \mathrm{~m}$ in diameter, and is still enlarging. Therefore, their final dimensions are not yet known. New dolines are still forming, almost twenty days after the main earthquake event.

Other than dropout dolines, the suffosion dolines are also registered (at the Mečenčani school backyard and in fields near the village), indicating the gradual subsidence of superficial coverbeds which form a funnel-shaped depression at the surface (Table 1). As opposed to dropout dolines, in this case the process advances from the surface, usually in noncohesive sediment, as the finer fraction tends to move into the cavity underneath, whereas the coarser fraction remains closer to the surface (KRANJC, 2013).

\subsubsection{Liquefaction}

Liquefaction is a phenomenon wherein a mass of soil loses a large percentage of its shear resistance and flows in a manner resembling a liquid. The basic cause responsible for the liquefaction of saturated sands is the build-up of excess pore water pressure due to either cyclic or shock loading of the sand (e.g. during an earthquake). If drainage cannot take place, then the tendency to compress the volume of the grains causes an increase in pore water pressure. In loose sands, pore water pressure can build up to the point where it is the same as the overburden pressure, causing complete loss of strength which initiates liquefaction. However, NORRIS et al. (1998) pointed out that a loose sand does not always lose all strength during liquefaction. Loose sands at low confining pressure, and medium and dense sands undergo only limited deformation due to dilation once initial liquefaction has occurred. Such a response is referred to as ranging from limited liquefaction (in the case of loose and medium dense sands at low confining pressure) to dilative behaviour (in dense sands) (BELL, 2007). Liquefaction is more likely to occur in sandy or non-plastic silty soils, but may in rare cases occur in gravels and clays. The increase of the portion of finer particles in the sand somewhat increases the resistance to liquefaction. Also, liquefaction is most commonly reported in the upper 12 to $15 \mathrm{~m}$ of the soil profile.

The liquefaction of subsurface layers in the research area is recognised by sand boiling phenomena. A sand boil is sand and water that erupts out onto the ground surface during an earthquake as a result of liquefaction at shallow depths (USGS Earth- quake Glossary, 2021). The research area exhibited many locations with such features, eighty-five are in the database at present (Table 2). Most of the sand boils are aligned in a row along a linear fracture exposed at the surface (Figure 5). Locations of sand boil phenomena are found in several areas adjacent to the rivers Sava, Kupa and Glina and are clear indications of liquefied zones.

Coseismic liquefaction in the research area also initiated lateral spreading, subsidence and river bank failures. The aforementioned processes are in some cases interrelated.

Lateral spreading is a type of movement that refers to movement of rock blocks or very coherent soil masses that rest on soft, deformable material (GONZÁLEZ DE VALLEJO \& FERRER, 2011). These movements are due to the loss of strength of the underlying material, which either flows or is deformed under the weight of the rigid blocks.

Here, the lateral spreading is the result of liquefaction of the saturated and loose sandy layers, and occurred on the flood embankments of the river Sava but also on the natural Kupa and Petrinjčica river banks (Figure 6). Horizontal displacements in most of the cases are from a few centimetres to several decimetres.

Subsidence caused by liquefaction is related to larger liquified zones and is registered at six sites. It is recognised or suspected in populated areas of Petrinja (Vinogradska and Milana Makanca street zones), Sisak and at the edges of smaller settlements Stari Brod, Letovanić, Brest, Drenčina and Palanjek. According to our knowledge, the vertical displacements in densely populated areas are up to 10 centimetres. Larger vertical displacements of several decimetres are noted in the immediate vicinity of the Kupa and Sava rivers (Figure 7).

\subsubsection{Landslides}

Landslide is a general term covering wide variety of mass-movement landforms and processes (including slides and rockfalls) involving the downslope transport of soil and rock material, under gravitational influence. Landslides can be triggered by earthquakes. According to KEEFER (1984) an earthquake with a Richter magnitude of 4 would probably not generate slides, but rockfalls and rock slides could be triggered by the weaker seismic tremors. 

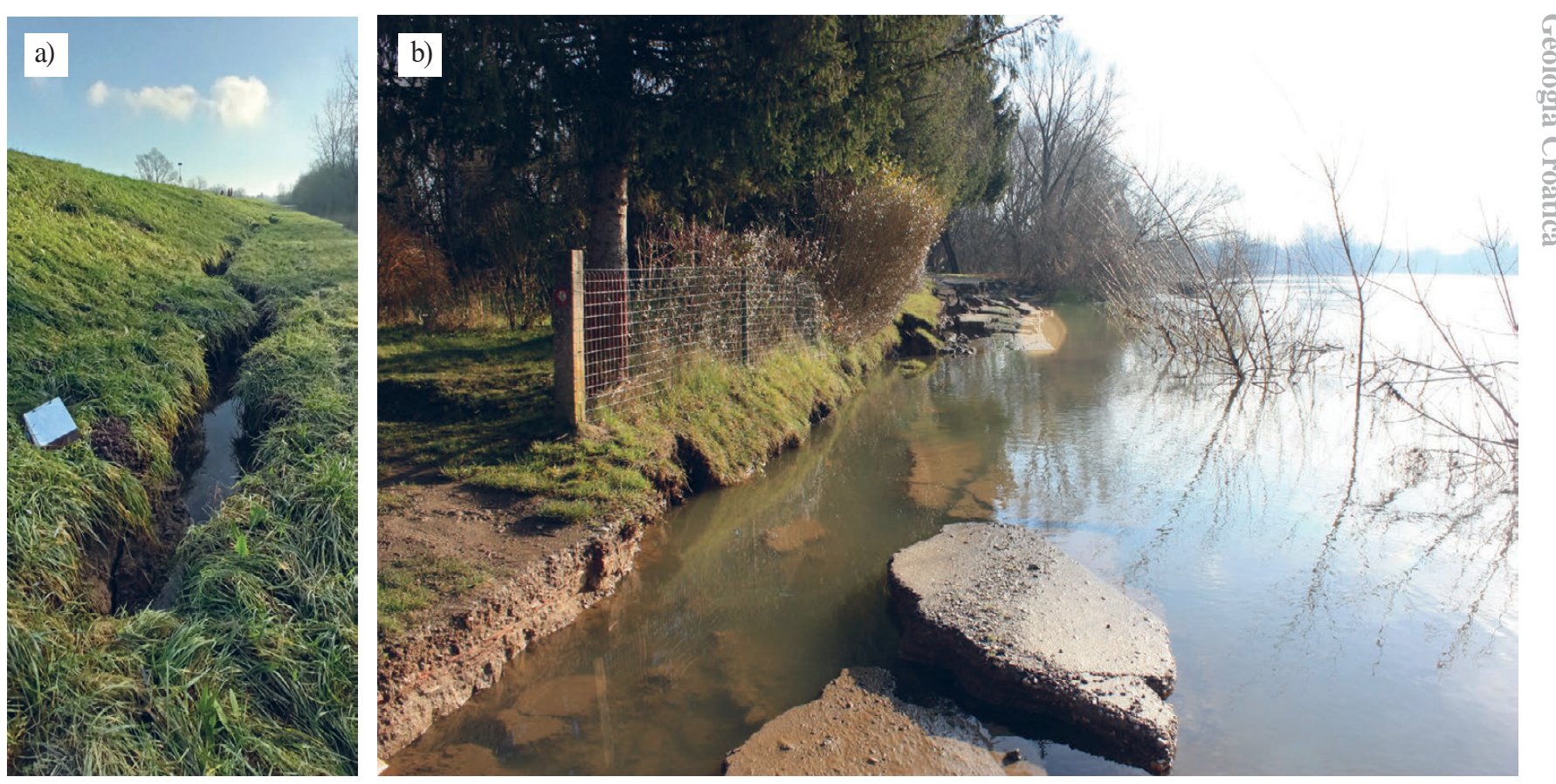

Figure 6. Lateral spreading and associated processes: a) failure of the flood embankment in Sisak (observation point Si-03); b) road failure in Letovanić (observation point RMT-35).

Slide is a landslide characterized by a shearing and rotary movement of a generally independent mass of rock or soil along the curved slip surface (concave upward) and about an axis parallel to the slope from which it descends (BATES \& JACKSON, 1980).

Rockfall is another type of landslide characterised by precipitous movement, free falling, bouncing or rolling, of a newly detached segment of bedrock of any size from a cliff or other very steep slope (BATES \& JACKSON, 1980; DORREN, 2003). Along the detachment surface, little or no shear displacement takes place (VARNES, 1978).

Thirty- six slides and four rockfalls have been registered so far, after the Petrinja 2020 earthquake. Most of the registered slides or rockfalls are older landslides reactivated by ground motion. Their nature and horizontal displacements vary greatly (Figure 8). It has been noted that even minor or not visible displacements, together with ground shaking could generate greater material damage on the structures located at the edge or inside the colluvium material. Some of the areas affected by the reacti- vation of landslides (mainly shallow ones), caused by the earthquake, are Hađer, Stari Farkašić, Petrinja and Sunja. Also, as already mentioned, in some cases slope failures are the consequence of liquefaction and lateral spreading. That is particularly the case in the zones where lateral support of the top layer is absent, dominantly along the river banks of the Kupa and Petrinjčica rivers (Figure 6).

Rockfalls have also been registered. Most of them are located below known rockfall source areas or steep slopes. Since rockfalls are very rapid to extremely rapid events, ground motions of this earthquake immediately triggered block motions causing rolling and bouncing of the blocks of various sizes into the foot of the slope (Figure 8b).

\subsection{Other ground effects}

Other registered coseismic ground effects will be shortly presented, because these effects still remain to be evaluated and interpreted in the near future. So far, the observed ground effects are preliminary differentiated according to the medium in which
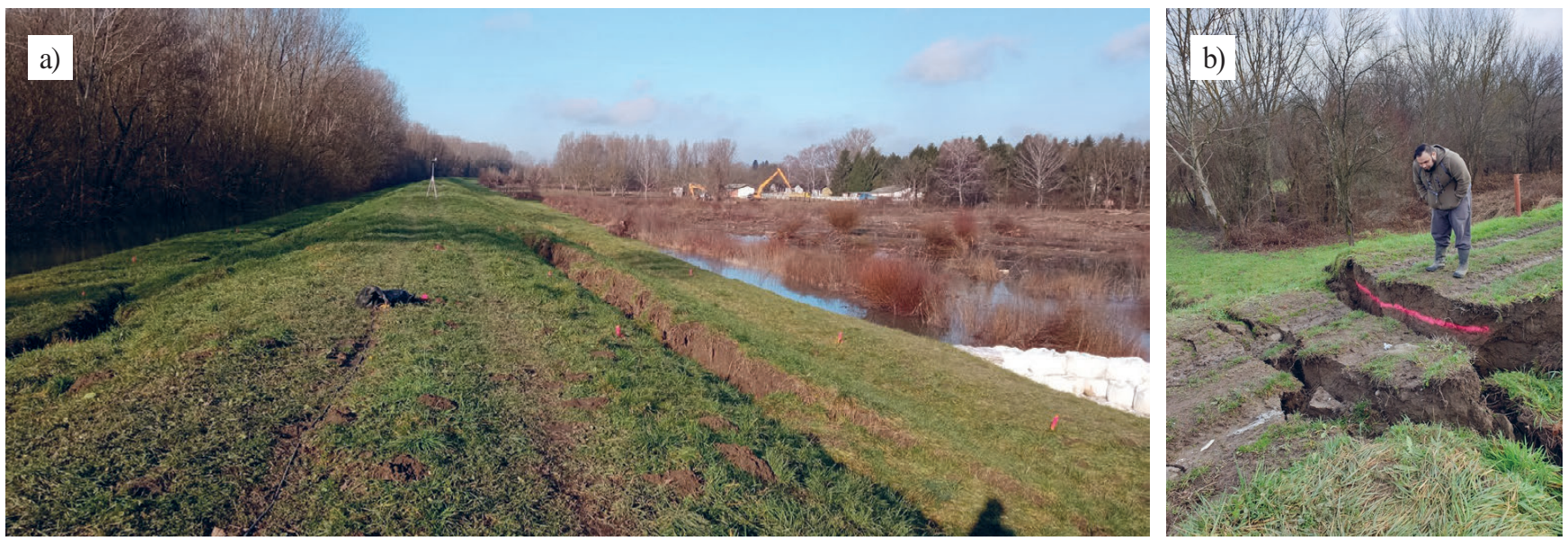

Figure 7. Subsidence and lateral spreading of the embankments near the Sava river: a) in Sisak (observation point Si-03); b) in Palanjek (observation point NP-21). 

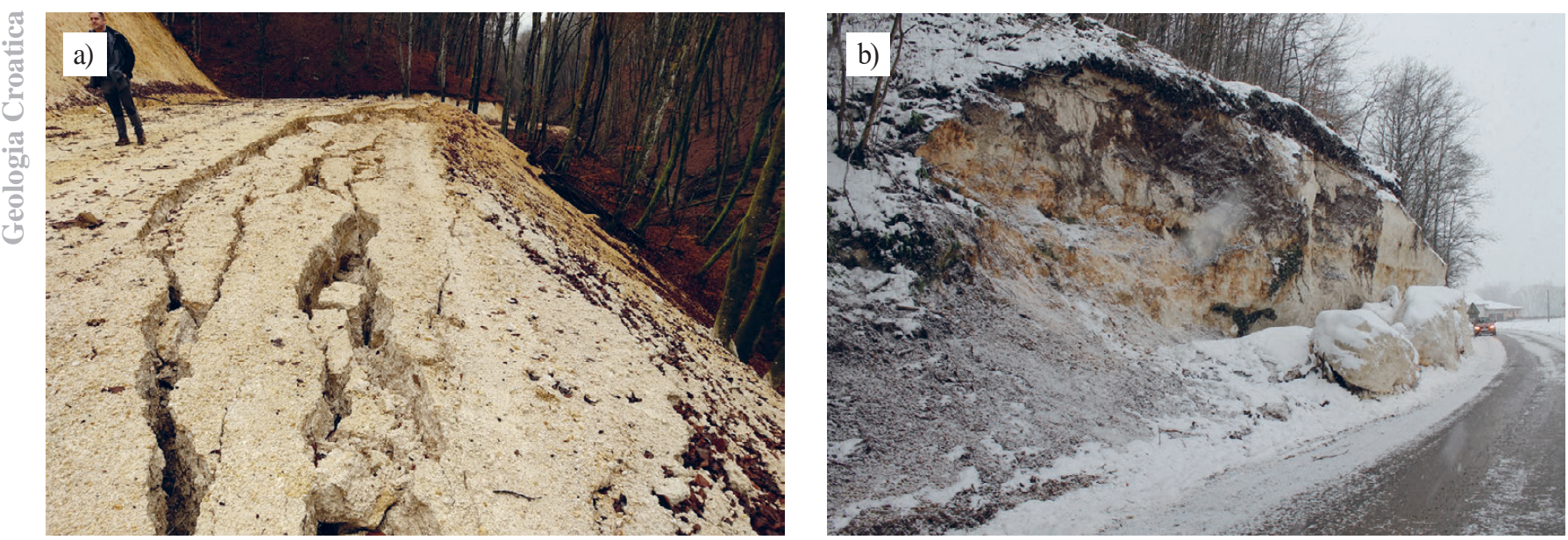

Figure 8. Coseismic landslide activation: a) slide in forest road embankment (near Strašnik, observation point ŠP-3); b) rockfall near Pecki that blocked the local road (observation point IK-ODR).

they are registered. Earthquake-induced ground effects in natural materials are classified as geological, while all other effects on man-made structures are considered as infrastructural (Table 1).

A number of soil ruptures are observed in various lithologies (Figure 2). In general, two types of soil ruptures can be differentiated. The first group comprises ruptures closely related to the main seismogenic fault zone that triggered the major earthquake. The second group comprises soil ruptures that are related to liquified zones and surface subsidence. Fracture displacements are usually up to a few centimetres, rarely several decimetres (Figure 9).

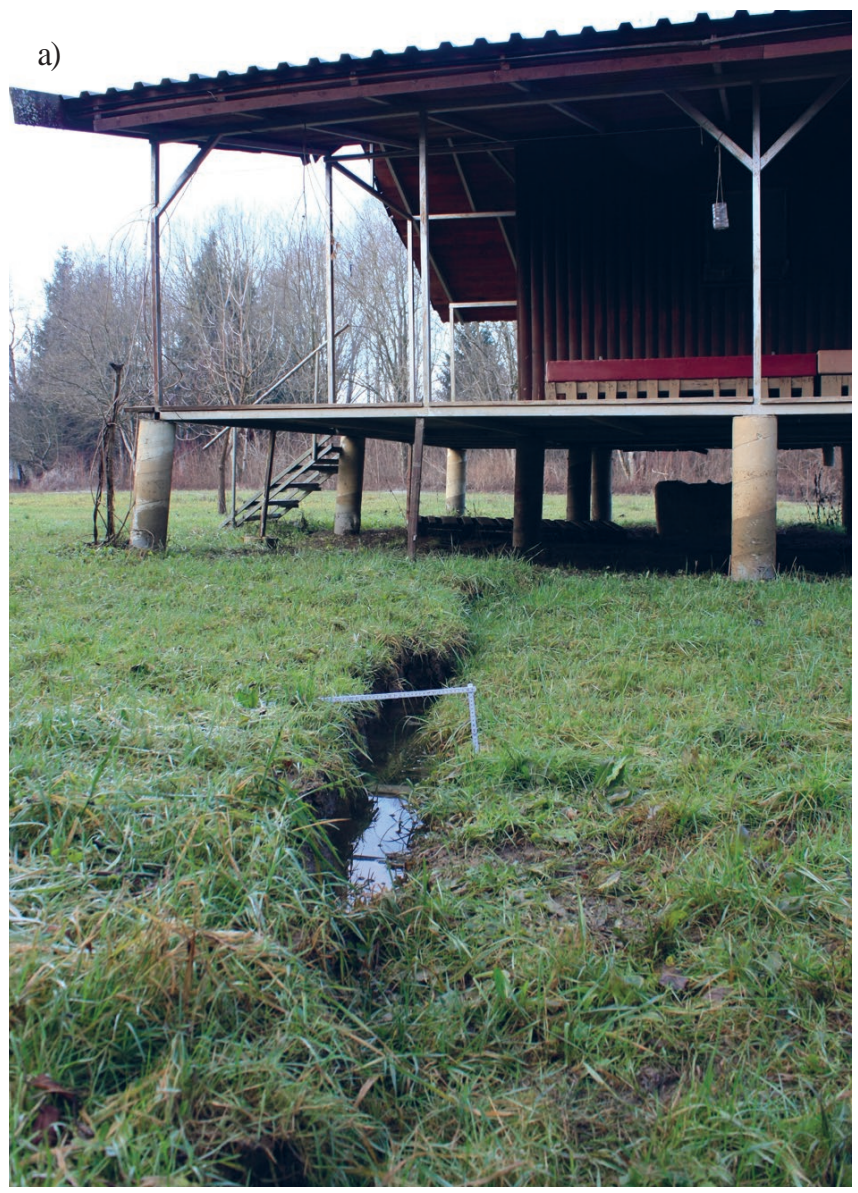

At a single location in Hrastovica, the displacement or clouding of springwater is registered. Here, before the main earthquake, the groundwater catchment collected all waters above the family house, but after intensive ground shaking, the groundwater emergence was scattered in a wider zone causing groundwater eruption inside and around the family house (Figure 10a).

Numerous road ruptures are also registered (Figure 10b). The great majority of these fractures are in the wider zone of the activated seismogenic fault (Figure 2). Other road ruptures follow minor, local faults, or are related to liquefaction-induced subsidence.

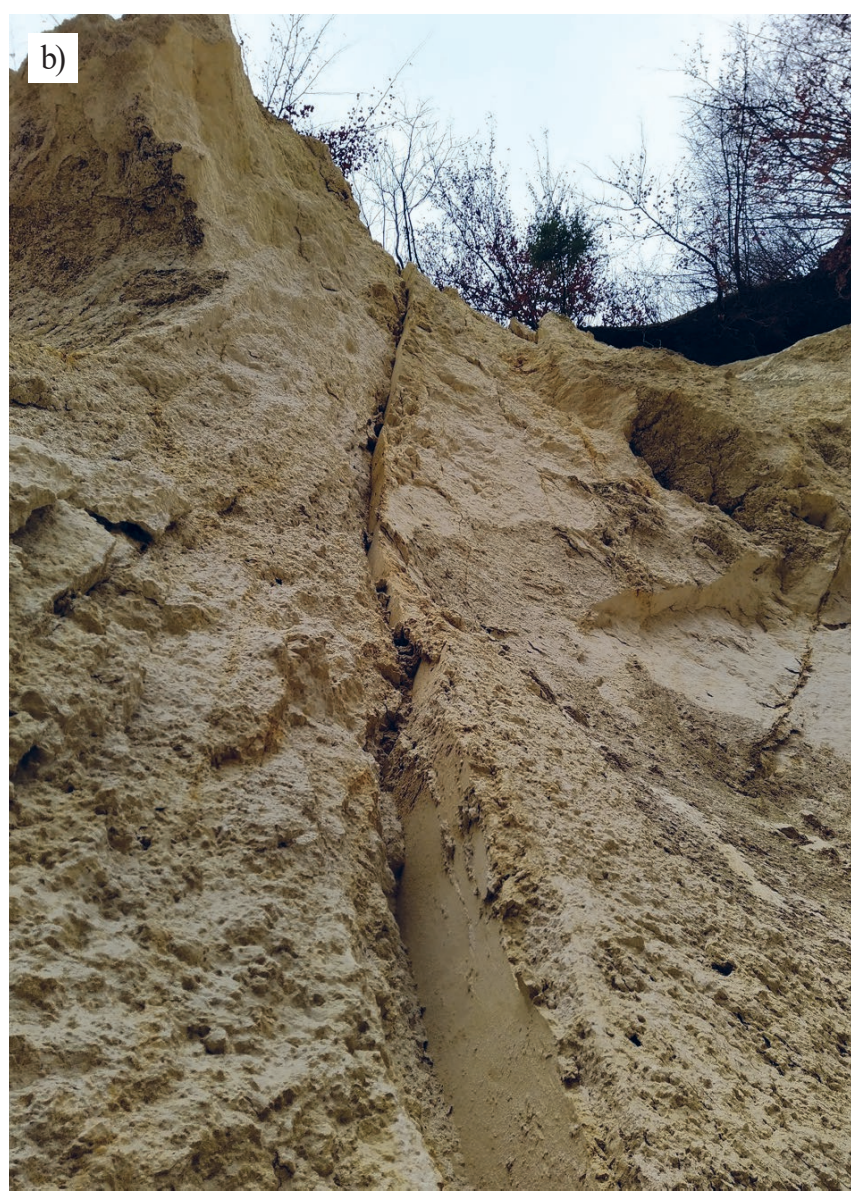

Figure 9. Soil rupture: a) near Letovanić (observation point RMT-38); b) near Župić village (observation point MV-11). 

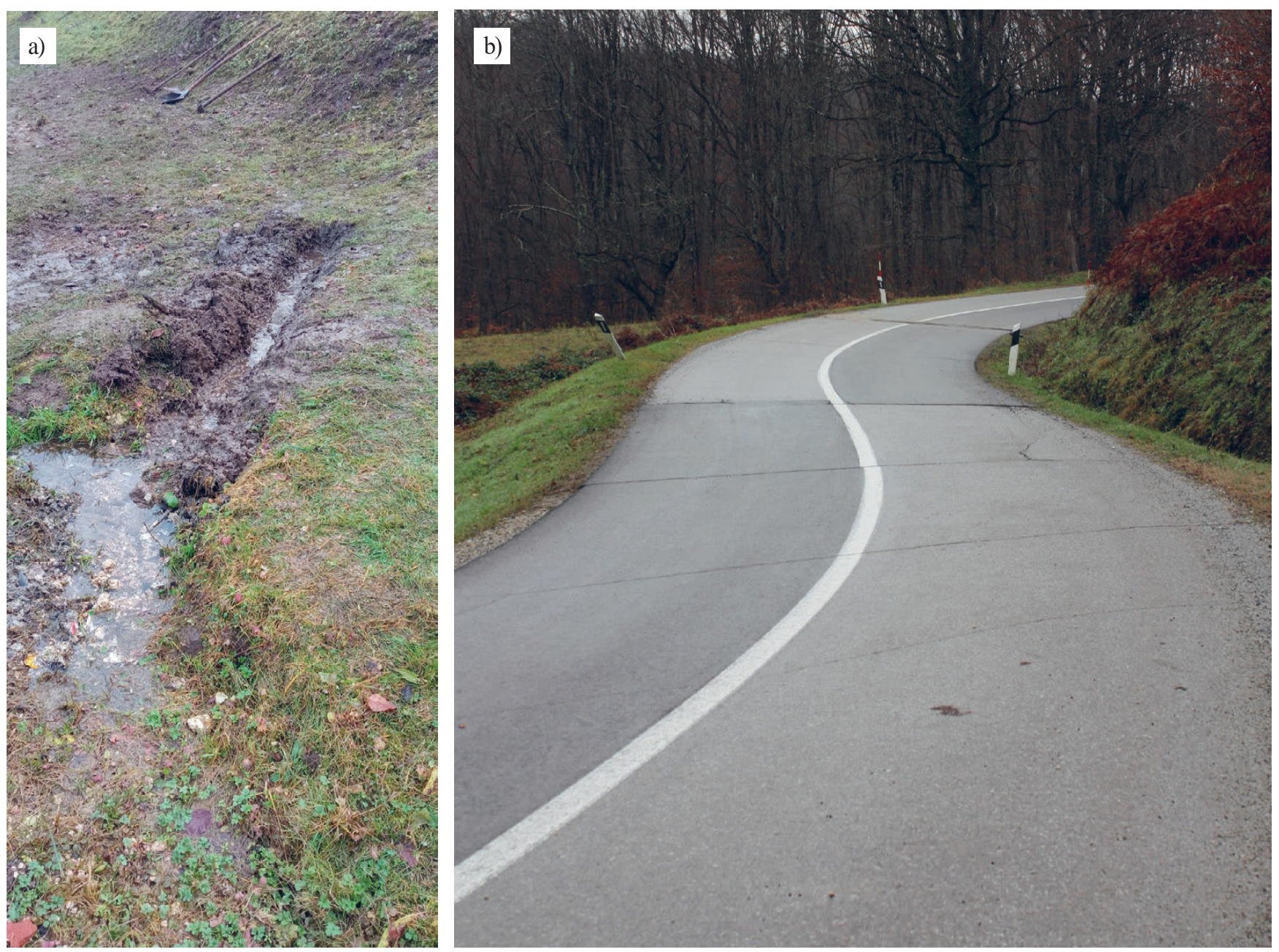

Figure 10. a) clouding of springwater in Hrastovica (observation point HR-DN-01); b) road ruptures near Pokupsko Vratečko (observation point RMT-43).

\section{DISCUSSION AND CONCLUSIONS}

As seen from the geological map, the locations of noted coseismic ground failures are strongly related to the geological setting and composition (Figure 2). Therefore, this chapter provides preliminary comments on the relationship between the geological setting and ground failures occurrences in the research area. In addition, details are presented about existing geological and other relevant data, together with recommendations for further explorations.

The spatial distribution of various types of coseismic ground failures/effects in the Petrinja earthquake seismic series is shown in Figure 2. A similar up to date map view presentation of the collected data, will be available in the form of WMS at the Croatian geological survey web portal (www.hgi-cgs.hr). Despite the fact that data collection for this publication was limited to only two weeks after the major December, $29^{\text {th }} 2020$ Petrinja earthquake, the spatial distribution of the recorded coseismic ground failures and their generating processes already indicate endangered areas. It is possible that further data collection will discover additional locations or areas affected by different post-seismic effects, but here, the comments and conclusions are related to the available information.

\subsection{Subsidence dolines area}

The subsidence dolines are exclusively found in the area around the villages of Mečenčani and Borojevići. This area was surveyed in detail during the hydrogeological investigations for Pašino vrelo (ŠIMUNOVIĆ \& HEĆIMOVIĆ, 1998; MRAZ, 2005; MRAZ et al., 2007; LARVA et al., 2010). The terrain is characterised by alluvial and proluvial sediments $\left(\right.$ al- $\left.\mathrm{Q}_{2}\right)$ which overlie Miocene rocks (Figure 11). Freshly exposed steep banks of newly formed dolines facilitate understanding of the nature of alluvial sediments which are soil mixtures of various fractions from clay to gravel. The thickness of these deposits varies from several to $\sim 15$ metres in the vicinity of the river Sunja. Older Miocene deposits $\left(\mathrm{M}_{3}\right)$ are represented by clayey sands and clays. They are overlain by $\mathrm{M}_{4}$ deposits, beginning with conglomerates or breccias followed by Lithothamnium limestones. According to geophysical investigations, those limestones are more than $100 \mathrm{~m}$ thick in some parts (Krsnik, 2005).

The Lithothamnium limestones represent the main aquifer for Pašino vrelo. The upper weathering zone in the limestone is from 10 to $25 \mathrm{~m}$ thick, with the frequent appearance of wide-open joints, smaller cavities and caverns (MRAZ, 2005). Groundwater level in this area is very shallow, from 1.2 to $2.0 \mathrm{~m}$. All of the factors are favourable for a good yield of the Pašino vrelo which is used for the public water supply. The Pašino vrelo is a natural spring, from an old dropout doline, with a short stream that flow toward the Sunja River. There are several older dolines in the area, and some of them are periodic rather than permanent springs. It is important to emphasize that groundwater extraction at the Pašino vrelo does not significantly influence the total groundwater flow in the area, and the well influence is limited to several tens of metres around it. The closest buildings in villages 


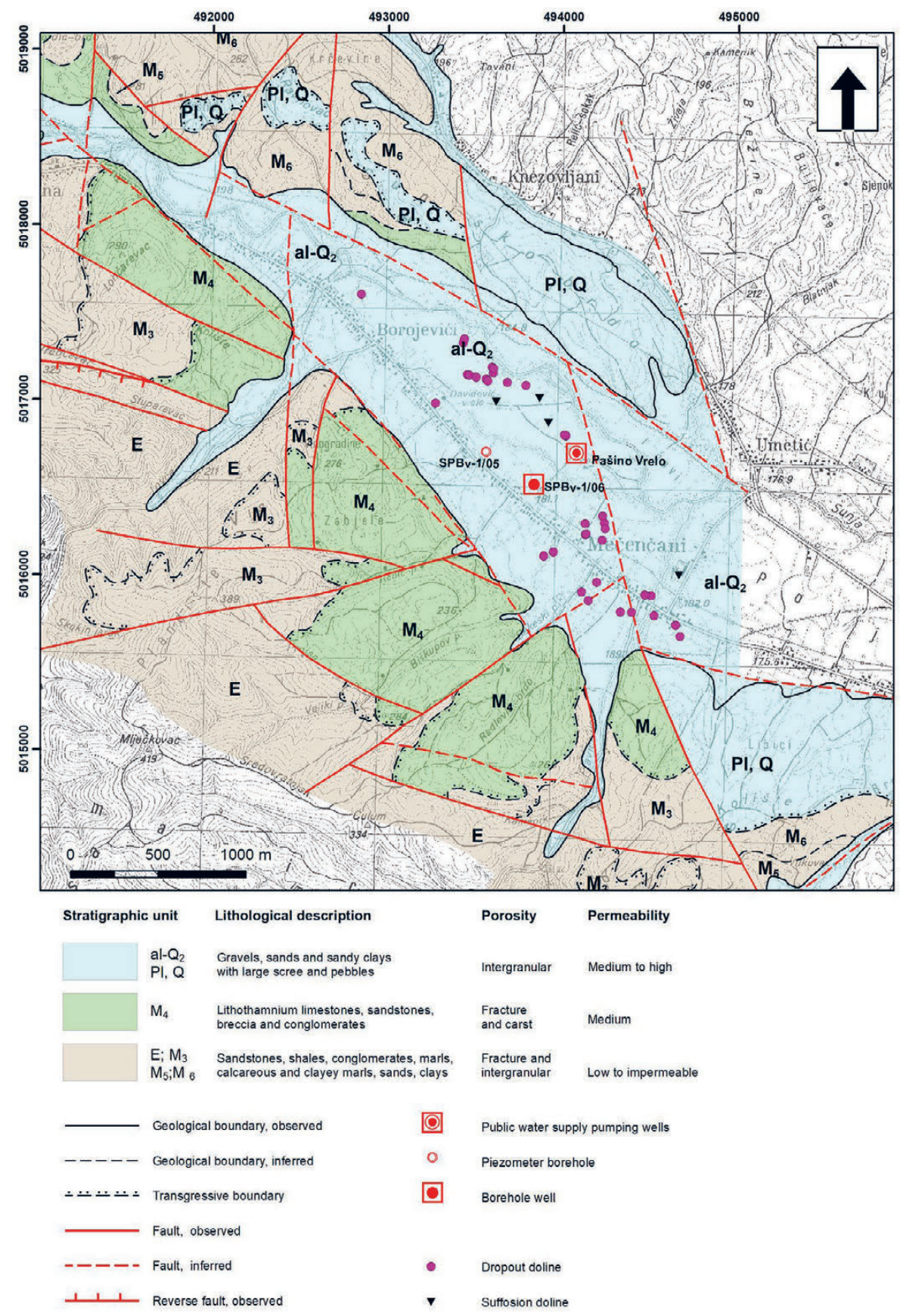

Figure 11. The distribution of subsidence dolines in the wider Mečenčani and Borojevići area on the geological map (originally in the scale 1:5 000; MRAZ, 2005).

are some $200 \mathrm{~m}$ from the spring. Since the aquifer is very karstified, the main task in forthcoming future research will be recognizing the main preferential flow paths typical for karst aquifers. Groundwater flow is one of the main causes for the development of dropout dolines and they occur mainly above these zones. This process happens regularly in this area, but over long periods of time. The Petrinja earthquake series significantly accelerated the collapse of several caverns and initiated the rapid development of new dolines, which represents a serious threat to the houses in this area.

Since some of the newly formed dolines are developing very closely to family houses and even below them, further detailed research is urgent. Firstly, remote sensing (with UAVs - unmanned aerial vehicles) of the wider Mečenčani and Borojevići area in order to determine the exact positions, sizes, and spatial distribution of the dolines was requested. Analysis of a detailed digital terrain model obtained by UAV data acquisition and the possible detection of newly formed surface depressions can govern the positioning of geophysical profiling and core drilling in order to define the depth of soil cover on top of the limestones, detect existing underground cavities and define their size, interrelationships and provide sampling from deeper horizons. Borehole in situ tests, installation of piezometer and laboratory analysis of the soil samples may also be needed in order to provide data for the detection of failure mechanisms, and the influence of seismic waves and ground water interaction causing rapid development of dolines in this area. It was suggested that site exploration covers the wider area in order to enable the production of a detailed subsidence susceptibility map.

In addition, monitoring of the area should be established. Monitoring should include periodic remote sensing, groundwater level monitoring and monitoring of ground shaking intensity. These data, with the results of the other aforementioned investi- 


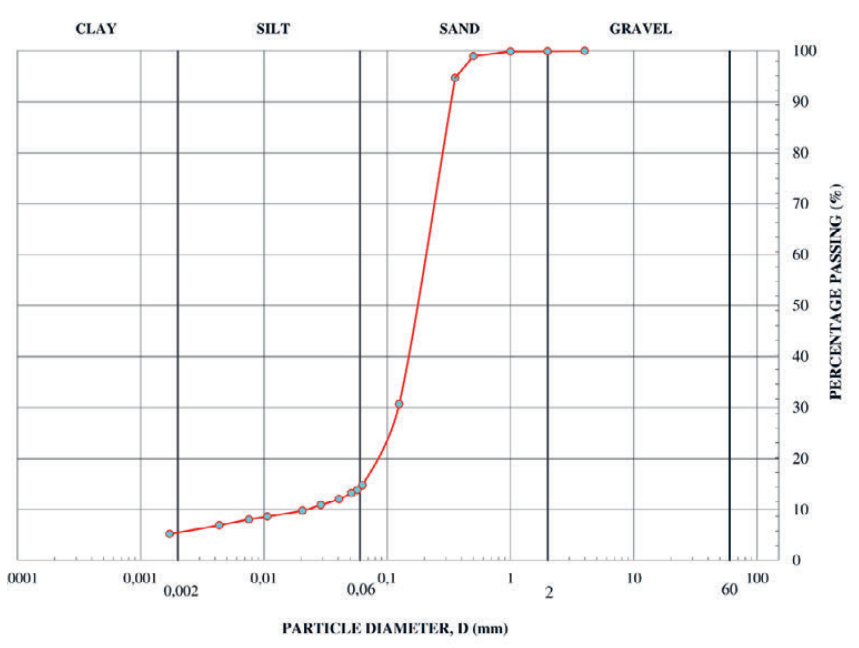

Figure 12. Granulometric diagram of sand boil material sampled at the surface in Brest Pokupski. The analysis is done in accordance with HRN EN ISO 178924:2016 norm.

gations, should provide insight into the rates of doline development in time and their relationship to ground conditions.

Suffosion processes, enlarging and forming new subsidence dolines in this area are still rapidly progressing, therefore research in this area should be given a high priority in dealing with geological hazards induced by earthquakes. Within the forthcoming winter and spring, when the hydrological maximum usually occurs in this area (peaks of both the precipitation and the groundwater levels), new dolines can be formed, especially if some stronger aftershocks occur when groundwater levels are high. Locations of the dolines suggest preferential flow paths in the karstic underground, and these zones will be the main target of geophysical profiling (electrical tomography and refraction seismic). These preferential flow paths in karst aquifers are usually caused by fault or fracture zones where the rock mass is highly karstified. Above these karstified rocks, suffosion of Quaternary deposits is significantly stronger than in other areas, so the combination of karstification and suffosion weakens these zones, while the earthquake accelerates the whole process. It is important to emphasize that groundwater level (pressure) in the karstic aquifer is usually higher than that in the Quaternary deposits overlying the aquifer, which also increases the suffosion at the soil/rock contact.

\subsection{Liquefied areas}

Liquefaction is mainly traced by boiling sand phenomena at the surface (Figure 5). According to the preliminary data, most of the phenomena are spotted in the recent alluvial sediments related to the rivers Sava, Kupa and Glina (Figure 2).

The alluvium of these recent rivers is dominated by pure sands, only locally by silts. Siltose or gravelly sands, and lenses of pure gravels are also observed (PIKIJA et al., 1986). The material ejected at the surface is predominantly classified as pure sand, which is confirmed by granulometry laboratory analysis (Figure 12).

Locally, liquefaction effects are registered in the flood sediments. These sediments are dominantly fine grained clayey and sandy silts or fine-grained sands (PIKIJA et al., 1986). Due to their depositional nature (sedimentation from an aqueous suspension after flooding), their thickness depends on the terrain configuration and is usually up to $5 \mathrm{~m}$.
The surface effects of liquefaction are not registered in the minor and thinner alluvium deposits of smaller rivers including the Petrinjčica, Moštanica and other streams (Figure 2). Namely, their alluvial sediments are dominated by gravels (PIKIJA et al., 1986), coarser sediments less susceptible to liquefaction.

The distances of the boiling sand occurrences from these rivers also confirms the strong relationship between liquefaction and the larger alluvial sedimentary bodies. Namely, distances measured from the aforementioned rivers are up to 1000 metres. The exception is the area of the Vinogradska street in Petrinja, where the liquefied area also involves Pliocene sediments in the base (clays, gravels and sands) (according to available geological map in the scale 1: 100 000). Another isolated case of possible liquefaction in Pliocene sediments is registered in Posavci near Kravarsko, situated far away from any significant river flow $(>8000$ $\mathrm{m})$. Therefore, for these reasons, the possibility of liquefaction of saturated sandy layers in Pliocene sediments should be studied in more detail.

The preliminary conclusion is that liquefaction almost exclusively occurred in widespread and thicker alluvial sediments containing significant beds or lenses of loose and "pure" sand layers.

Considering all the collected data in the research area, favourable conditions for liquefaction are related to the Sava, Kupa and Glina alluvium or floodplain sediments which, according to rough and preliminary estimations cover more than $100 \mathrm{~km}^{2}$ (Figure 13). The presented map is based solely on current database records and existing geological data at the scale of 1:100.000 and should be considered as a rough estimation. Detailed studies are needed in order to define liquefaction susceptibility zones and mitigation measures.

As can be seen, liquefaction-induced lateral spreading and ground subsidence can cause considerable damage to civil infrastructure. In this area, lateral spreads frequently occurred on the fairly flat area alongside streams or river courses causing river bank failures. Ground subsidence usually happened in the near vicinity of the river courses where loose sandy layers are of greater thickness and are completely saturated with water. This resulted in the settling of houses, buckling of roads, and subsidence of flood embankments. It is important to note that the settlement and tilting of some buildings is also observed in the postseismic period and even though some of these are marked as 'usable' from a structural point of view. Their behaviour is strongly affected by the ground conditions and can lead to state limits being exceeded.

It is important to point out that the occurrence of liquefaction in some layers is not necessarily associated with the damage of structures. If a thick layer of non-liquefiable soil exists near the ground surface, and if the thickness of the underlying layer of liquefied sand is low, then the damaging effects of liquefaction will not be manifested at the ground surface (ISHIHARA, 1985). Still, considering great potential for damage as a consequence of liquefaction and its related processes, it is suggested that more detailed engineering geological and geotechnical exploration is performed. The exploration should cover the whole Croatian region that meets liquefaction-favourable conditions and are also zones of high seismicity. The aim of such a study should be the mitigation of, and preparedness for liquefaction-induced damage.

There are many international researchers who have studied liquefaction potential and defined liquefaction-induced hazards. Maps showing the degree of liquefaction susceptibility are based on the likely response of subsurface materials to seismic loading 


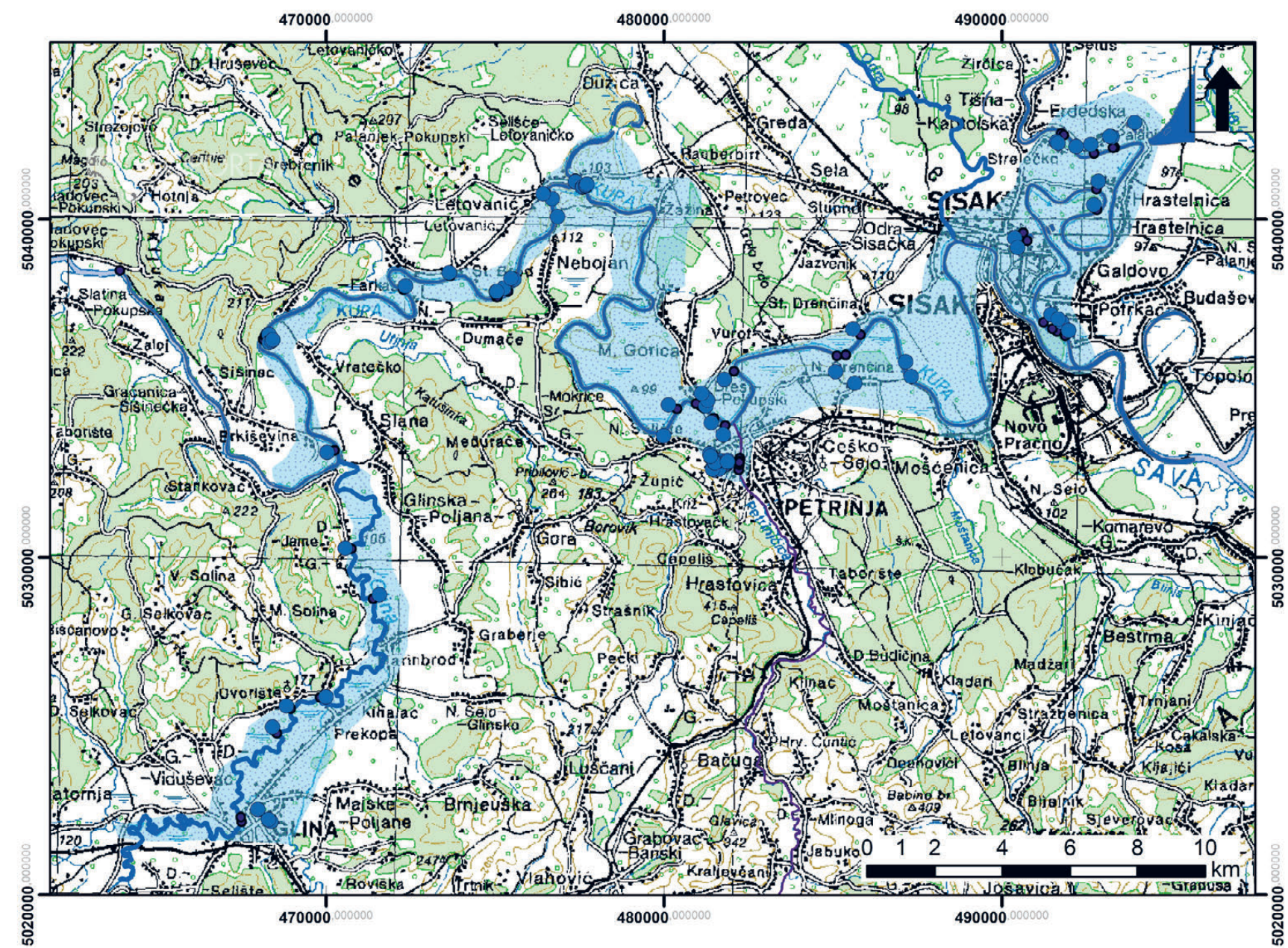

Figure 13. Rough and preliminary estimations of the area with favourable conditions for liquefaction processes (shaded blue). The estimation is based on recent surface processes caused by liquefaction (blue dots) and existing Basic Geological Map in the scale 1: 100.000.

(YOUD \& PERKINS, 1987; KAJIHARA et al., 2016). A study about the liquefaction potential of sediments in the Zagreb area was presented by VEINOVIĆ et al. (2007), who also authored a doctoral thesis entitled Evaluation of possibility for liquefaction susceptibility and setting bases for liquefaction zonation in Croatia (VEINOVIĆ, 2007), which could be a good basis for starting research in potentially endangered zones.

\subsection{Landslides}

Landslide locations are scattered across the research area but are mostly related to particular lithologies (Figure 14), being predominantly observed in Pl and Pl-Q sediments. Both units are characterised by clays, sands and gravels, and are well known for frequent landslides in the much wider region. Several landslides in loess sediments are recognised here, which are also observed in the wider Kutina area. Landslides on top of "rocky" $\mathrm{M}_{5}, \mathrm{M}_{4}$ and Pc-E units are probably related to soil material in their weathering zones.

The alluvial (a, ap) and diluvial (dpr) units are presented separately, because sliding processes on flat areas in these sediments are frequently related to liquefaction processes and lateral spreading along river banks, causing river bank failure (Figure 14).

Factors to be considered in assessing the potential for earthquake-induced landslides, according to GONZÁLEZ DE VALLEJO \& FERRER (2011) are the presence of unstable slopes or slopes in precarious states of stability prior to the earthquake, steep slopes, soils with low strength or metastable structures (quick clays, collapsible soils) and rock scarps with potential rockfall hazards. Therefore, in estimations of coseismic landslide susceptibility, besides the usual parameters, the aforementioned additional factors should be considered. The authors' opinion is that due to different geological medium and failure mechanisms, sliding and falling susceptibility should be analysed separately.

As for other susceptibility maps, landslide susceptibility analysis should start with inventory. The reliable inventories are based on visual analysis of remote sensing derivates. Besides, appropriate to the scale/details in which the map is prepared, a relevant geological map is essential. Depending on the specific properties of the area in focus, additional factors and data are combined in order to produce a reliable landslide susceptibility map. Until now in Croatia, various landslide susceptibility maps at different scales were prepared by different engineering-geologists, but for

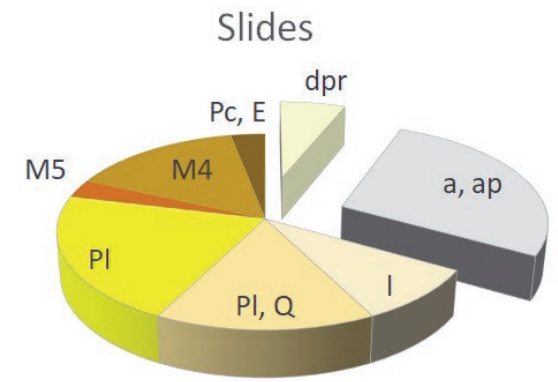

Figure 14. The proportional distribution of landslides in particular geological units. 
this region three maps are relevant. For the whole Croatian territory, a small-scale landslide susceptibility map displays general susceptibility for large regions (PODOLSZKI et al., 2014; ERAK, 2018).

Regional sliding susceptibility map for the whole of SisakMoslavina County at the scale of 1: 100.000 has been prepared for the safEarth project (BOSTJANČIĆ et al. 2021).

Rockfall susceptibility maps consider different input factors. For a regional approach, the rockfall inventory is essential. The analysis of large areas subjected to such processes is based on the identification of the rockfall source areas using remote sensing techniques. A detailed bare earth digital terrain model (DTM) obtained by LIDAR can help in defining rockfall source areas. Using a detailed (1:5.000) geological map and the aforementioned remote sensing based inventory, it is possible to produce a reliable rockfall susceptibility map (TOŠEVSKI, 2018). Such a map should indicate zones endangered by falling rocks triggered by intensive precipitation or earthquakes. The procedure for a more detailed rockfall threat assessment, for specific locations, require much more data and field work, and is described in BOSTJANČIĆ \& POLLAK (2020).

Earthquakes and related natural geohazard processes cannot be prevented, but their effects on construction can be mitigated. Therefore, the focus of geological experts is directed toward hazard identification and preventive measures. In order to undertake that successfully, systematic research and extensive studies of various geological hazards are essential. Geological hazards are studied in different projects and their results are applied locally, but central inventories of the geohazard processes are not supported. National, open and sustainable databases are basic preconditions for the proper evaluation of geological hazards for the whole Croatian territory. Therefore, the initiative by the HGI (www.hgi-cgs.hr) for their existence and sustainability will hopefully be reinforced by responsible agencies in order to allow their growth and use.

\section{REFERENCES}

AVANIĆ, R., KOVAČIĆ, M., PAVELIĆ, D. \& PEH, Z. (2018a): The Neogene of Hrvatsko Zagorje.- In: TIBLJAŠ, D., HORVAT, M., TOMAŠIĆ, N., MILEUSNIĆ, M. \& GRIZELJ, A. (eds.): 9th Mid-European Clay Conference, Conference book, Field Trip Guide book, Zagreb, 128-131.

AVANIĆ, R., ŠIMUNIĆ, A. \& PEH, Z. (2018b): Geology of the Croatian Zagorje Region.- In: RMAN, N., MARKOVIĆ, T. \& BRENČIĆ, M. (eds.): 5. Slovenski geološki kongres, Post congress field trip book, Ljubljana, p. 35.

BAČIĆ, M., IVŠIĆ, T. \& KOVAČEVIĆ, M.S. (2020): Geotechnics as an unavoidable segment of earthquake engineering.- Građevinar, 72/10, 923-936, doi: 10.14256/ JCE.2968.2020

BADA, G., HORVÁTH, F., DÖVÉNYI, P., SZAFIAN, P., WINDHOFFER, G. \& CLOETINGH, S. (2007): Present-day stress field and tectonic inversion in the Pannonian basin.- Global and Planetary Change, 58, 165-180. doi: 10.1016/j.gloplacha.2007.01.007

BASILI, R., KASTELIC, V., DEMIRCIOGLU, M.B., GARCIA MORENO, D., NEMSER, E.S., PETRICCA, P., SBORAS, S.P., BESANA-OSTMAN, G.M., CABRAL, J., CAMELBEECK, T., CAPUTO, R., DANCIU, L., DOMAC, H., FONSECA, J., GARCÍA-MAYORDOMO, J., GIARDINI, D., GLAVATOVIC, B., GULEN, L., INCE, Y., PAVLIDES, S., SESETYAN, K., TARABUSI, G., TIBERTI, M.M., UTKUCU, M., VALENSISE, G., VANNESTE, K., VILANOVA, S. \& WÖSSNER, J. (2013): The European Database of Seismogenic Faults (EDSF) compiled in the framework of the Project SHARE. http://diss.rm.ingv.it/share-edsf/SHARE_ WP3.2 Database.html, doi: 10.6092/INGV.IT-SHARE-EDSF.

BATES, R.L. \& JACKSON, J.A. (1980): Glossary of Geology American Geological Institute (AGI).- Falls Church, $751 \mathrm{p}$.

BELL, F.G. (2007): Engineering Geology, Second Edition.- Elsevier Ltd., 575 p.

BIRD, J.F. \& BOMMER, J.J. (2004): Earthquake losses due to ground failure.- Engineering Geology, 75/1, 147-179. doi: 10.1016/j.enggeo.2004.05.006

BOMMER, J.J. \& RODRÍGUEZ, C.E. (2002): Earthquake-induced landslides in Central America, Eng. Geol., 63, 189-220. doi: 10.1016/S0013-7952(01)00081-3
BOSTJANČIĆ, I. \& POLLAK, D. (2020): Rockfall threat assessment along railways in carbonate rocks in Croatia.- Bulletin of Engineering Geology and the Environment, 79, 3921-3942. doi: 10.1007/s10064-020-01822-x.

BOSTJANČIĆ, I., FILIPOVIĆ, M., GULAM, V. \& POLLAK, D. (2021): Regional-Scale Landslide Susceptibility Mapping Using Limited LiDAR-Based Landslide Inventories for Sisak-Moslavina County, Croatia. Sustainability, 13, 4543. https://doi. org $/ 10.3390 /$ su 13084543

CAPRARI, P., DELLA SETA, M., MARTINO, S., FANTINI, A., FIORUCCI, M. \& PRIORE, T. (2018): Upgrade of the CEDIT database of earthquake-induced ground effects in Italy.- Italian Journal of Engineering Geology and Environment, 2, 23-39. doi: 10.4408/IJEGE.2018-02.O-02

DORREN, L.K.A. (2003): A review of rockfall mechanics and modelling approaches.Progress in Physical Geography: Earth and Environment, 27/1, 69-87. doi: 10.1191/0309133303pp359ra.

ERAK, M. (2018): Karta podložnosti na klizanje Republike Hrvatske [Landslide susceptibility map of the Republic of Croatia - in Croatian].- Unpubl. Master's thesis. Faculty of Mining, Geology and Petroleum Engineering, University of Zagreb, Zagreb, $35 \mathrm{p}$

GONZÁLEZ DE VALLEJO, L.I. \& FERRER, M. (2011): Geological engineering.-CRC Press/Balkema, 678 p. doi: 10.1201/b11745

GORUM, T., FAN, X., VAN WESTEN, C.J., HUANG, R.Q., XU, Q., TANG, C. \& WANG, G. (2011): Distribution pattern of earthquake - induced landslides triggered by the 12 May 2008 Wenchuan earthquake.- Geomorphology, 133, 3-4, 152-167. doi: 10.1016/j.geomorph.2010.12.030

GOVORČIN, M., HERAK, M., MATOŠ, B., PRIBIČEVIĆ, B. \& VLAHOVIĆ, I. (2020): Constraints on Complex Faulting during the 1996 Ston-Slano (Croatia) Earthquake Inferred from the DInSAR, Seismological, and Geological Observations.- Remote Sensing, 12, 1157. doi: 10.3390/rs12071157

GRIZELJ, A. (2004): Mineraloške i geokemijske značajke gornjomiocenskih pelitnih sedimenata jugozapadnog dijela Hrvatskog zagorja [Mineralogical and geochemical characteristics of Upper Miocene pelitic sediments of the south-western part of Hrvatsko zagorje - in Croatian].- Unpubl. Master's thesis, Faculty of Science, University of Zagreb, Zagreb, $118 \mathrm{p}$.

HALAMIĆ, J., BELAK, M., PAVELIĆ, D., AVANIĆ, R., FILJAK, R., ŠPARICA, M., BRKIĆ, M., KOVAČIĆ, M., VRSALJKO, D., BANAK, A. \& CRNKO, J. (2019): Osnovna geološka karta Republike Hrvatske mjerila $1: 50.000$ - Požeška gora [Basic Geological Map of the Republic of Croatia 1:50.000 - Požeška gora - in Croatian].- Croatian Geological Survey, Department of Geology, Zagreb.

HERAK, D., HERAK, M. \& TOMLJENOVIĆ, B. (2009): Seismicity and earthquake focal mechanisms in NorthWestern Croatia.- Tectonophysics, 465, 212-220. doi: 10.1016/j.tecto.2008.12.005

HERAK, D., HERAK, M., PRELOGOVIĆ, E., MARKUŠIĆ, S. \& MARKULIN, Ž. (2005): Jabuka island (Central Adriatic Sea) earthquakes of 2003.- Tectonophysics, 398, 167-180. doi: 10.1016/j.tecto.2005.01.007

HERAK, D., HERAK, M. \& VRKIĆ, I. (2017a): Velika trešnja, seizmičnost i potresna opasnost na širem dubrovačkom području [Great earthquake, seismicity and seismic hazard in the wider Dubrovnik area - in Croatian].- Dubrovnik: časopis za književnost i znanost XXVIII, 1, 5-18.

HERAK, D., SOVIĆ, I., CECIĆ, I., ŽIVČIĆ, M., DASOVIĆ, I. \& HERAK, M. (2017b): Historical seismicity of the Rijeka region (NW External Dinarides, Croatia) - Part I: Earthquakes of 1750, 1838 and 1904 in the Bakar epicentral area.- Seismological Research Letters, 88, 904-915.

HERAK, D., ŽIVČIĆ, M., VRKIĆ, I. \& HERAK, M. (2020): The Međimurje (Croatia) Earthquake of 1738.- Seismological Research Letters, 91, 1042-1056. doi: $10.1785 / 0220190304$

HERAK, M., ALLEGRETTI, I., HERAK, D., IVANČIĆ, I., KUK, V., MARIĆ , K., MARKUŠIĆ , S. \& SOVIĆ , I. (2011): Republika Hrvatska, Karta potresnih područja [Republic of Croatia, Seismic Hazard Maps - in Croatian].- University of Zagreb, Faculty of Science, Department of Geophysics. http://seizkarta.gfz.hr/ karta.php. Accessed 25 January 2021. In: Eurocode 8: design of structures for earthquake resistance-part 1: general rules, seismic actions and rules for buildings. National Annex.- Croatian Standards Institute, p. 28.

ISHIHARA, K. (1985): Stability of natural deposits during earthquakes.- In: Proceedings of the Eleventh International Conference on Soil Mechanics and Foundation Engineering, San Francisco, 12-16 August, 321-377.

KAJIHARA, K., POKHREL, R.M., KIYOTA, T. \& KONAGAI, K. (2016): Liquefaction-induced ground subsidence extracted from Digital Surface Models and its application to hazard map of Urayasu city, Japan.- In: The 15th Asian Regional Conference on Soil Mechanics and Geotechnical Engineering, Japanese Geotechnical Society Special Publication, 829-834. doi: 10.3208/jgssp.TC203-02.

KEEFER, D.K. (1984): Landslides caused by earthquakes.- Bulletin of the Geological Society of America 95, 406-421.

KOVAČIĆ, M. (2004): Sedimentologija gornjomiocenskih naslaga jugozapadnog dijela Panonskog bazena. [Sedimentology of the Upper Miocene deposits from the southwest part of the Pannonian Basin - in Croatian].- Unpubl. PhD Thesis, Faculty of Science, University of Zagreb, 203 p.

KRANJC, A. (2013): Classification of closed depressions in carbonate karst.- In: SHRODER, J. (Editor in Chief), FRUMKIN, A. (ed.): Treatise on Geomorphol- 
ogy. Academic Press, San Diego, CA, Vol. 6, Karst Geomorphology, 104-111. doi: 10.1016/B978-0-12-374739-6.00125-1

KRSNIK, M. (2005): Geofizička istraživanja područja Pašinog vrela [Geophysical research of the Pašino vrelo spring area - in Croatian].- Unpubl. Technical report, Archive IGH d.d., U 0255/05.

KUREČIĆ, T. (2017): Sedimentologija i paleoekologija Pliocenskih Viviparus slojeva Vukomeričkih Gorica [Sedimentology and paleoecology of Pliocene Viviparus beds from the area of Vukomeričke Gorice - in Croatian, with an English Abstract].- Unpubl. PhD Thesis, Faculty of Science, University of Zagreb, $168 \mathrm{p}$.

KUREČIĆ, T., KOVAČIĆ, M. \& GRIZELJ, A. (submitted): Mineral assemblage and provenance of the pliocene Viviparus Beds from the area of Vukomeričke gorice (Central Croatia).- Geologia Croatica.

KVASNIČKA, P. \& MATEŠIĆ, L. (2001): Geotechnical data base for the City of Zagreb and its application in site response analyses.- In: Fourth International Conference on Recent Advances in Geotechnical Earthquake Engineering and Soil Dynamics, San Diego, California, 11-15.

KVASNIČKA, P., MATEŠIĆ, L. \& SKRAČIĆ, S. (1998): Geotehnička podloga grada Zagreba. [Geotechnical basis of the city of Zagreb - in Croatian].- Građevinar, $50 / 1,119-127$

LARVA, O., MARKOVIĆ, T. \& MRAZ, V. (2010): Hydrodynamic and hydrochemical conditions at the groundwater source "Pašino vrelo", with focus on its development.- Geologia Croatica, 63/3, 299-312. doi:104154/gc.2010.24

MAJER, V. \& LUGOVIĆ, B. (1985): Metamorfne stijene u ofiolitnoj zoni Banije, Jugoslavija. II. Amfiboliti (metabaziti) [Metamorphic rocks in the Banija ophiolite zone, Yugoslavia: II.Amphibolites (metabasites) - in Croatian].-Acta Geologica JAZU, 15/2, 25-49, Zagreb.

MAJER, V. (1984): Metamorfne stijene u ofiolitskoj zoni Banije, Jugoslavija. I. Metapeliti [Metamorphic rocks in the Banija ophiolite zone of Banija, Yugoslavia. I. Metapelites - in Croatian].- Rad JAZU, 41/1, 35-82, Zagreb.

MANDIC, O., KUREČIĆ, T., NEUBAUER, T.A. \& HARZHAUSER, M. (2015): Stratigraphic and paleogeographic significance of lacustrine mollusks from the Pliocene Viviparus beds in central Croatia.- Geologia Croatica, 68/3, 179-207. doi: $10.4154 / G C .2015 .15$

MARKUŠIĆ, S., STANKO, D., KORBAR, T., BELIĆ, N., PENAVA, D. \& KORDIĆ, B. (2020): The Zagreb (Croatia) M5.5 Earthquake on 22 March 2020.- Geosciences, 10, 252. doi: 10.3390/geosciences 10070252

MARTINO, S., PRESTININZI, A. \& ROMEO, R.W. (2014): Earthquake-induced ground failures in Italy from a reviewed database.- Natural Hazards and Earth System Sciences, 14, 799-814. doi: 10.5194/nhess-14-799-2014

MATEŠIĆ, L. \& KVASNIČKA, P. (2007): Geotechnical data management according to ISO 9001.- In: CUELLAR et al. (eds): Proceedings of the 14th European conference on soil mechanics and geotechnical engineering, Madrid. Mill press science publishers, 1715-1719.

MATEŠIĆ, L., KVASNIČKA, P. \& MIHALIĆ, S. (2011): Importance of data and process management in Eurocode 7.- Geofizika, 28/1, 99-107.

MRAZ, V. (2005): Vodoistražni radovi „Pašino vrelo“ [Hydrogeological investigations of the Pašino vrelo spring - in Croatian].- Unpubl. Technical report, Archive of the Croatian Geological Survey, 88/05.

MRAZ, V., LARVA, O. \& MARKOVIĆ, T. (2007): Izvorište „Pašino vrelo“. Zaštitne zone za novi zdenac SPB PV - 01/06 i vodocrpilište „Pašino vrelo“. [Pašino vrelo spring - sanitary protection zones for the new extraction well SPB PV-01/06 and the spring itself - In Croatian].- Unpubl. Technical report, Archive of the Croatian Geological Survey, 64/07.

NORRIS, G., GAHIR, Z. \& SIDDHARTHAN, R. (1998): An effective stress understanding of liquefaction behaviour-- Environmental and Engineering Geoscience, 4, 93-101.

PAMIĆ, J. (2002): The Sava-Vardar Zone of the Dinarides and Hellenides versus the Vardar Ocean.- Eclogae Geologicae Helvetiae, 95, 99-113.

PAVELIĆ, D. \& KOVAČIĆ, M. (2018): Sedimentology and stratigraphy of the Neogene rift-type North Croatian Basin (Pannonian Basin System, Croatia): A review.- Mar. Petrol. Geol., 91, 455-469. doi: 10.1016/j.marpetgeo.2018.01.026

PIKIJA, M. (1986): Osnovna geološka karta SFRJ 1:100.000. Tumač za list Sisak. [Basic Geological Map of SFRY 1:100.000, Geology of the Sisak sheet - in Croatian].Institut za geološka istraživanja, Zagreb, Savezni geol. zavod, Beograd, 55 p.

PIKIJA, M. (1987): Osnovna geološka karta SFRJ 1:100.000 list Sisak [Basic Geological Map of SFRY 1:100.000, Sisak sheet - in Croatian].- Geol. Zavod, Zagreb, Savezni geol. Zavod, Beograd.

PODOLSZKI, L., POLLAK, D., GULAM, V. \& MIKLIN, Ž. (2014): Development of Landslide Susceptibility Map of Croatia.- In: LOLLINO G. et al. (eds.): Engineering Geology for Society and Territory - Volume 2, 947-950. doi: 10.1007/978-3319-09057-3 164

PRESTININZI, A. \& ROMEO, R. (2000): Earthquake-induced ground failures in Italy.Engineering Geology, 58/3-4, 387-397. doi: 10.1016/S0013-7952(00)00044-2

RAFFAELLI, P. \& MAGDALENIĆ Z. (1970): Metamorphic and magmatic rocks in the Gvozdansko-Brezovo Polje area. Banija.- Bull. Sci. Cons. Acad. Yougosl., (A), Zagreb, 15/9-10, 313-314.

RODRÍGUEZ, C.E., BOMMER, J.J. \& CHANDLER, R.J. (1999): Earthquake-induced landslides: 1980-1997.--Soil Dynamics and Earthquake Engineering, 18, 325-346. doi: 10.1016/S0267-7261(99)00012-3
SAFTIĆ, B., VELIĆ, J., SZTANÓ, O., JUHÁSZ, G. \& IVKOVIĆ, Ž. (2003): Tertiary Subsurface Facies, Source Rocks and Hydrocarbon Reservoirs in the SW Part of the Pannonian Basin (Northern Croatia and South-Western Hungary).- Geologia Croatica, 56/1, 101-122.

ŠAVOR NOVAK, M., UROŠ, M., ATALIĆ J., HERAK, M., DEMŠIĆ, M., BANIČEK, M., LAZAREVIĆ, D., BIJELIĆ, N., CRNOGORAC, M. \& TODORIĆ, M. (2020): Zagreb earthquake of 22 March 2020 - preliminary report on seismologic aspects and damage to buildings.- Građevinar, 72, 10, 843-867

SCHMID, S.M., FÜGENSCHUH, B., KOUNOV, A., MATENCO, L., NIEVERGELT, P., OBERHÄNSLI, R., PLEUGER, J., SCHEFER, S., SCHUSTER, R., TOMLJENOVIĆ, B., USTASZEWSKI, K. \& VAN HINSBERGEN, D.J. (2020): Tectonic units of the Alpine collision zone between Eastern Alps and Western Turkey.- Gondwana Research, 78, 308-374. doi: 10.1016/j.gr.2019.07.005

SCHMITT, R.G., TANYAS, HAKAN, NOWICKI, JESSEE, M.A., ZHU, JING, BIEGEL, K.M., ALLSTADT, K.E., JIBSON, R.W., THOMPSON, E.M., VAN WESTEN, C.J., SATO, H.P., WALD, D.J., GODT, J.W., GORUM, TOLGA., XU, CHONG, RATHJE, E.M. \& KNUDSEN, K.L. (2017): An open repository of earthquake-triggered ground-failure inventories.- U.S. Geological Survey data release collection, data series 1064, accessed June 26. doi: 10.5066/F7H70DB4

ŠIKIĆ, K. (2014a): Osnovna geološka karta RH 1:100.000, list Bosanski Novi [Basic Geological Map of Republic Croatia 1:100.000, Bosanski Novi sheet - In Croatian].- Croatian Geological Survey, Zagreb.

ŠIKIĆ, K. (2014b): Osnovna geološka karta RH 1:100.000, Tumač za list Bosanski Novi [Basic Geological Map of Republic Croatia 1:100.000, Geology of the Bosanski Novi sheet - In Croatian].- Croatian Geological Survey, Zagreb, 111 p.

ŠIMUNOVIĆ, A. \& HEĆIMOVIĆ, I. (1998): Geološka i hidrogeološka istraživanja bliže okolice Pašinog vrela [Geological and hydrogeological study in the vicinity of "Pašino vrelo"- in Croatian].- Unpubl. Technical report, Archive of the Croatian Geological Survey, 88/98

TOMLJENOVIĆ, B. \& CSONTOS, L. (2001): Neogene-Quaternary structures in the border zone between Alps, Dinarides and Pannonian Basin (Hrvatsko zagorje and Karlovac basins, Croatia).- International Journal of Earth Sciences, 90, 560-578. doi: $10.1007 / \mathrm{s} 005310000176$

TORBAR, J. (1882): Izvješće o Zagrebačkom potresu [The report on Zagreb earthquake - In Croatian]. - JAZU, Zagreb, 141 p.

TOŠEVSKI,A. (2018): Podložnost porječja rijeke Dubračine površinskim geodinamičkim procesima [Susceptibility of the Dubracina river basin to the superficial geodynamical processes - in Croatian].- Unpubl. PhD Theses, Faculty of Mining, Geology and Petroleum Engineering, University of Zagreb, $251 \mathrm{p}$.

U.S. Geological Survey (2021): Earthquake Glossary at URL: https://earthquake.usgs. gov/learn/glossary/

USTASZEWSKI, K., HERAK, M., TOMLJENOVIĆ, B., HERAK, D. \& MATEJ, S. (2014): Neotectonics of the Dinarides-Pannonian Basin transition and possible earthquake sources in the Banja Luka epicentral area.- Journal of Geodynamics, 82, 52-68. doi: 10.1016/j.jog.2014.04.006

USTASZEWSKI, K., KOUNOV, A., SCHMID, S.M., SCHALTEGGER, U., KRENN, E., FRANK, W. \& FÜGENSCHUH, B. (2010): Evolution of the Adria-Europe plate boundary in the northern Dinarides: From continent-continent collision to back-arc extension.- Tectonics, 29, TC 6017, 1-34. doi: 10.1029/2010TC002668

USTASZEWSKI, K., SCHMID, S.M., LUGOVIĆ, B., SCHUSTER, R., SCHALTEGGER, U., BERNOULLI, D., HOTTINGER, L., KOUNOV, A., FÜGENSCHUH, B. \& SCHEFER, S. (2009): Late Cretaceous intra oceanic magmatism in the internal Dinarides (northern Bosnia and Herzegovina): Implications for the collision of the Adriatic and European plates.- Lithos, 108, 106-125. doi: 10.1016/j.lithos.2008.09.010

VARNES, D.J. (1978): Slope movement types and processes.- In: SCHUSTER, R.L. \& KRIZEK, R.J. (eds): Landslides: analysis and control, Vol. 176. National Academy of Sciences, TRB special report, 11-33.

VEINOVIĆ, Ž. (2007): Ocjena mogućnosti pojave likvefakcije i definiranje osnove za likvefakcijsko zoniranje na teritoriju Republike Hrvatske. [Assessment of the possibility of liquefaction occurrence and defining the basis for liquefaction zoning on the territory of the Republic of Croatia - in Croatian].- Unpubl. PhD Theses, Faculty of Mining, Geology and Petroleum Engineering, University of Zagreb, $330 \mathrm{p}$.

VEINOVIĆ, Ž., DOMITROVIĆ, D. \& LOVRIĆ, T. (2007): Pojava likvefakcije na području Zagreba u prošlosti i procjena mogućnosti ponovne pojave tijekom jačeg potresa [Historical occurrence of liquefaction in Zagreb area and estimation of reoccurrence in case of another strong earthquake - in Croatian].- Rudarskogeološko-naftni zbornik, 19, 111-120.

WALTHAM, A.C. \& FOOKES, P.G. (2003): Engineering classification of karst ground conditions.- Quarterly Journal of Engineering Geology and Hydrogeology, 36, 101-118. doi: 10.1144/1470-9236/2002-33

WILLIAMS, P. (2004): Dolines.- In: GUNN, J. (ed.): Encyclopedia of Caves and Karst Science.- Fitzroy Dearborn, New York, NY, and London, 304-310.

YOUD, T.L. \& HOOSE, S.N. (1978): Historic ground failures in northern California associated with earthquakes.- USGS Professional Paper, 993, 177 p.

YOUD, T.L. \& PERKINS, J.B. (1987): Map I-127-G.- US Geological Survey, Washington, DC. 